\title{
MATHEMATICAL THEORY OF SINGLE CHANNEL SYSTEMS. ANALYTICITY OF SCATTERING MATRIX
}

\author{
BY
}

I. M. SIGAL

\begin{abstract}
ABSTRACr. We show that the $S$-matrix of a quantum many-body, short-range, single-channel system has a meromorphic continuation whose poles occur at most at the dilation-analytic resonances [28], [24] and at the eigenvalues of the Hamiltonian. In passing, we prove the main spectral theorem (on location of the essential spectrum) and asymptotic completeness for the mentioned class of systems.
\end{abstract}

Introduction. In this paper we study the analytic properties of the scattering matrix for many-body, short-range, single-channel systems. Our main theorem asserts that the scattering matrix has a meromorphic continuation in the energy parameter into a certain sector of the complex plane. The poles of this continuation occur only at eigenvalues of the dilation analytic family $H(\zeta)$ associated with the Hamiltonian $\boldsymbol{H}$. By Balslev's and Combes' theorem the latter eigenvalues lead to the poles of the meromorphic continuation of the matrix elements $\left\langle\phi,(H-z)^{-1} F\right\rangle$ on the dilation analytic vectors across the continuous spectrum into the second Riemann sheet. Moreover, if the potentials are nice enough so that the negative axis belongs to the meromorphic domain, then the poles on this axis occur at most at the negative eigenvalues of $H$.

To prepare the ground for the proof of our main theorem we prove most of the theorems of the spectral and scattering theory for single-channel systems. These results are not new, but some of the theorems (e.g. the asymptotic completeness) contain improvements over the previous results.

Most of the work is done in an abstract setting and only at the end we check the assumptions of the abstract theorems. This allows us to treat the problems of different character separately. The abstract approach has forced us to introduce the notions of the abstract Schrödinger operator and abstract many-body system. We hope they will be useful in extending our methods to other systems.

The paper is self-contained; all necessary definitions are given in the text. $\S \S I-V$ contain the preparatory abstract results; the main theorem is formulated and proved in $\S \mathrm{VI}$, where all relevant definitions and preliminary results for $N$-body systems are also given. §§VII and VIII deal with the estimates of certain operators built out of the potentials and the free resolvent. These estimates are needed in the theorem of $\S \mathrm{VI}$ on the behavior of $(H-z)^{-1}$ near the continuous spectrum. In

Received by the editors June 26, 1980 and, in revised form, January 19, 1981.

1980 Mathematics Subject Classification. Primary 81C99, 35J10, 35P05; Secondary 45A10, 57F05, $81 \mathrm{C99}$.

() 1982 American Mathematical Society 0002-9947/81/0000-1061/\$08.25 
§IX we prove the existence of the meromorphic continuations for the wave operators. Generalizations, different approaches and literature are discussed in $\S X$. The appendix contains some abstract results about the regularizers needed in the main text.

ACKNOWLEDGEMENT. On the early stage of the work on this paper the author has benefited from discussions with E. Balslev, J.-M. Combes, K. Hepp and W. Hunziker, to whom he is very grateful. The author is also very grateful to B. Simon for important remarks on the manuscript, to $\mathrm{K}$. Sinha for pointing out an inaccuracy in the preprint, and to a referee for carefully reading the manuscript and making many useful remarks which led to improvement of the paper.

I. Abstract Schrödinger operators spectral theory. Let $T$ be a selfadjoint operator on a Hilbert space $\mathcal{H}$ and $\mathbb{Q}$ a finite lattice. Let, furthermore, $\left\{V_{a}, a \in \mathbb{Q}\right\}$ be the collection of $T$-bounded operators with the relative bounds 0 . The following operators are well defined on $D(T)$ :

$$
H=T+\sum V_{a} \text { and } H_{a}=T+\sum_{b \subset a} V_{b}
$$

If $V_{a}$ are symmetric then $H$ and $H_{a}$ are selfadjoint. We call $H$ an abstract Schrödinger operator (ASO). Set

$$
a_{\max }=\max \{a, a \in \mathbb{Q}\} \text { and } a_{\min }=\min \{a, a \in \mathbb{Q}\} .
$$

In this paper $b \subset a$ stands for the strict inclusion only.

Spectral theory of ASO.

Proposition I.1. Assume $V_{a} \exp (i T t) \stackrel{s}{\rightarrow} 0$ on some dense set from $D(T)$. Then $\sigma(T) \subset \sigma(H)$.

Proof. Let $\lambda \in \sigma(T)$ and $\left\{u_{n}\right\} \subset D(T)$ be such that $\left\|u_{n}\right\|=1$ and $\left\|(\lambda-T) u_{n}\right\|$ $\rightarrow 0(n \rightarrow 0)$. We have

$$
\left\|(\lambda-H) e^{i T_{t}} u_{n}\right\| \leqslant\left\|(\lambda-T) u_{n}\right\|+\left\|(H-T) e^{i T t_{n}} u_{n}\right\| \rightarrow 0
$$

as $n \rightarrow \infty$ and $|t| \rightarrow \infty$, which implies that $\lambda \in \sigma(H)$.

THEOREM I.2. Let the following condition be satisfied:

(A) $\Pi_{\cup a-a_{\max }}\left[V_{a}(T-z)^{-1}\right]$ is compact for some (and therefore for all) $z \in \rho(T)$ and any collection of $a$ 's, $\cup a=a_{\max }$. Then

$$
\sigma_{\text {ess }}(H) \subset \bigcup_{a \neq a_{\max }} \sigma\left(H_{a}\right) .
$$

A proof of the theorem will be given in §II.

Condition (A) is discussed in the following:

LEMMA I.3. Let there exist bounded operators $X_{a}^{n}, a \in \mathbb{Q}, n \in \mathrm{N}^{+}$, on $\mathcal{H}$ such that for $a, b \in \mathbb{Q}\left(R_{0}(z)=(T-z)^{-1}\right)$ :

(i) $V_{a} X_{a}^{n} R_{0}(i) \rightarrow 0(n \rightarrow \infty)$;

(ii) $\left[X_{a}^{n}, T\right] R_{0}(i) \rightarrow 0(n \rightarrow \infty)$;

(iii) $\left[X_{a}^{n}, V_{b}\right] R_{0}(i) \rightarrow 0(n \rightarrow \infty)$; 
(iv) $\Pi_{c}\left(\bar{X}_{c}^{n}\right) V_{b} R_{0}(i)$ is compact for any $n \in \mathrm{N}^{+}$whenever the c's satisfy $\cup c=$ $a_{\max }$. Here $\bar{X}_{a}^{n}=1-X_{a}^{n}$.

Then $\Pi\left[V_{a} R_{0}\right]$ is compact for any $z \in \cap \rho(T)$ and any collections of $a$ 's satisfying $\cup a=a_{\max }$.

PRoof. We claim that $\Pi \bar{X}_{a}^{(n)} \Pi\left[V_{a} R_{0}\right]$ converge in norm to $\Pi\left[V_{a} R_{0}\right]$ as $n \rightarrow \infty$. Then since the former operators are compact for $\cup a=a_{\max }$ in virtue of (iv), the compactness of the latter will follow from the theorem on closedness of the set of compact operator in the uniform topology. The convergence is proved in two steps. First we note that because of (i), $\Pi\left[V_{a} \bar{X}_{a}^{n} R_{0}\right]$ converge in norm to $\Pi\left[V_{a} R_{0}\right]$ as $n \rightarrow \infty$. On the second step we commute the $\bar{X}_{a}^{n}$ 's in the former operator to the left in the position in front of the first $R_{0}$ on the left. Because of the equation

$$
\left[\bar{X}_{a}^{n}, R_{0}\right]=R_{0}\left[X_{a}^{n}, T\right] R_{0}
$$

and conditions (ii) and (iii), the terms containing at least one commutator $\left[\bar{X}_{a}^{n}, R_{0}\right]$ or $\left[\bar{X}_{a}^{n}, V_{b}\right]$ vanish in norm as $n \rightarrow \infty$. Therefore the difference between the operators $\Pi\left[V_{a} \bar{X}_{a}^{n} R_{0}\right]$ and $\Pi \bar{X}_{a}^{n} \Pi\left[V_{a} R_{0}\right]$ goes in norm to zero as $n \rightarrow \infty$. This completes the proof.

COROLlary I.4. If an ASO $H$ satisfies the conditions of Lemma I.3, then $\sigma_{\text {ess }}(H) \subset \cup_{a \neq a_{\max }} \sigma\left(H_{a}\right)$.

Remark I.5. Theorem I.2 can be slightly generalized in order to fit our later considerations. Namely, condition (A) can be replaced by the requirement that $\Pi\left[B_{a_{1}} R_{0} A_{a_{1+1}}\right]$ are compact for some factorization $V_{a}=A_{a} B_{a}$. The factorization $A_{a}=1$ and therefore $B_{a}=V_{a}$ gives Theorem I.1. The factorization we use in the scattering theory is $A_{a}=\left|V_{a}\right|^{1 / 2}$.

II. Regularizers for $H-z$. Let $\mathcal{H}$ be a Banach space and $T$ an operator on $\mathcal{H}$ with a domain $D(T)$. We call a bounded operator $F$ from $\mathcal{H}$ to $D(T)$ a (right, exact) regularizer for $T$ iff (i) $F$ is invertible and (ii) $T F-1$, raised to some power, is compact on $\mathcal{H}$. In this section we construct regularizers for the family $H-z$ and use them to study the spectral properties of $H$.

Defintion II.1. Let $\mathcal{Q}$ be a finite lattice and $\left\{H_{a}, a \in \mathbb{Q}\right\}$, the collection of operators on $\mathcal{H}$ with $\mathscr{D}\left(H_{0}\right) \subset D\left(H_{a}\right)$, where $H_{0}=H_{a_{\min }}$. We define by induction on $a \in \mathbb{Q}$ the following families of bounded operators on $\mathcal{K}$ :

$$
A_{a}(z)=\left(H_{a}-z\right)\left(H_{0}-z\right)^{-1} \prod_{b \subset a} A_{b}(z)^{-1},
$$

where the arrow over the top of the product sign indicates the following order of the $A^{-1}$ 's: if $A_{c}^{-1}$ stands on the right of $A_{d}^{-1}$ then $c \notin d$.

We set the family of bounded operators from $\mathcal{H}$ to $D\left(H_{0}\right)$ :

$$
F_{a}(z)=\left(H_{0}-z\right)^{-1} \prod_{b \subset a} A_{b}(z)^{-1} .
$$

(II.1) and (II.2) imply that

$$
\left(H_{a}-z\right) F_{a}(z)=A_{a}(z)
$$


The obvious properties of $A_{a}$ and $F_{a}$ are listed for reference convenience in the following two lemmas:

LeMMA II.2. For any $a \in \mathbb{Q}$ and all $z \in \cap_{b \subset a} \rho\left(H_{b}\right)$, the operator $F_{a}(z)$ is bounded from $\mathcal{H}$ to $D\left(H_{0}\right)$ and has the bounded inverse (from $D\left(H_{0}\right)$ to $\left.\mathcal{H}\right)$. Both operators are analytic in $z \in \cap_{b \subset a} \rho\left(H_{b}\right)$.

LEMMA II.3. For any $a \in \mathbb{Q}$ and all $z \in \bigcap_{b \subset a} \rho\left(H_{b}\right)$, the operator $A_{a}(z)$ is bounded on $\mathcal{H}$ and is analytic in $z \in \cap_{b \subset a} \rho\left(H_{b}\right)$. It has the bounded inverse for $z \in \cap_{b \subseteq a} \rho\left(H_{b}\right)$ and the following statements are equivalent:

(1) $0 \in \sigma\left(A_{a}(z)\right)$ and $\varphi \in \operatorname{Ker} A_{a}(z)$;

(2) $z \in \sigma_{p}\left(H_{a}\right)$ and $F(z) \varphi \in \operatorname{Ker}(H-z)$.

In the case when the $H_{a}$ 's are constructed as in (I.1), the operators $F_{a}(z)$ and $A_{a}(z)$ have an additional structure:

LEMMA II.4. The operators $F_{a}(z)$ and $A_{a}(z)-1$ are finite, linear combinations of monomials of the form

$$
R_{0} \prod\left[V_{c} R_{b}\right], \quad c, b \subset a, \text { and } \Pi\left[V_{c} R_{b}\right], \quad b \subset a, \cup c=a,
$$

respectively. Here $R_{0}(z)=\left(H_{0}-z\right)^{-1}\left(H_{0}=T\right)$.

The statement can easily be derived by induction. The details can be found in [17]. Note here only that since $V_{a}$ have $T$-bounded 0 , they are $H_{b}$-bounded as well. Therefore monomials of form (II.4) are bounded and analytic in $z \in \cap \rho\left(H_{b}\right)$.

LEMMA II.5. For $z$ with $\operatorname{dist}(z, \sigma(T))$ sufficiently large, $A_{a}(z)-1$ is a norm convergent series of a-connected graphs $\Pi_{\cup c=a}\left[V_{c}(T-z)^{-1}\right]$.

Proof. The statement follows from Lemma II.4 and the fact that for $\operatorname{dist}(z, \sigma(T))$ large enough the following series are norm convergent:

$$
R_{b}(z)=(T-z)^{-1} \sum_{n=0}^{\infty}\left[\sum_{c \subset b} V_{c}(T-z)^{-1}\right]^{n} \text {. }
$$

Indeed, $\left\|A(T-z)^{-1}\right\| \rightarrow 0$ as $\operatorname{dist}(z, \sigma(T)) \rightarrow \infty$ for any $T$-bounded operator $A$.

In what follows we drop the subindex $a$ whenever $a=a_{\max }$.

Proof of Theorem I.2. We begin with

LEMMA II.6. The operator $A(z)-1$ is compact on $\mathcal{H}$ for all $z \in \cap_{a \neq a_{\max }} \rho\left(H_{a}\right)$.

Proof. The statement follows from Lemmas II.3 and II.5, condition (A) and the theorem about analytic families of compact operators (see e.g. [15]).

Lemmas II.2 and II.6 imply (see Theorem A.1) the statement of the theorem.

REMARK II.7. Lemmas II.2, II.3 and II.6 show that $F(z)$ is a regularizer for $H-z$.

III. Review of the Hilbert space scattering theory. In this section $H$ and $T$ are selfadjoint operators on a Hilbert space $\mathcal{H}, E_{p}$ is the projection on the subspace of 
the point spectrum, $\sigma_{p}(H)$, of $H$, and $E(\Delta)$ is the spectral projection for $H$. The strong limits

$$
W^{ \pm}=\underset{t \rightarrow \pm \infty}{s-\lim _{t \rightarrow \infty}} e^{i H t} e^{-i T t},
$$

whenever they exist on the absolute continuous subspace of $T$, are called the wave operators for the pair $(H, T)$. In order not to carry an extra symbol we assume henceforth that $T$ is absolute continuous. The scattering operator is defined on $\mathcal{H}$ by

$$
S=W^{+*} W^{-} \text {. }
$$

It commutes with $T,[S, T]=0$, and therefore is decomposable on a representation of $\mathcal{H}$ as a fiber direct integral $\int^{\oplus} \mathcal{H}_{\lambda} d \lambda$ with respect to $T: \Pi S \Pi^{*}=\int^{\oplus} S(\lambda) d \lambda$. Here $S(\lambda)$, called the scattering matrix, is an operator on $\mathcal{H}_{\lambda}$, and $\Pi$ is a unitary operator from $\mathcal{H}$ to $\int^{\oplus} \mathcal{H}_{\lambda} d \lambda$.

The definition of $W^{ \pm}$implies (1) $W^{ \pm}$are isometric, $W^{ \pm *} W^{ \pm}=1$, (2) $W^{ \pm}$are intertwining operators for $(H, T), H W^{ \pm}=W^{ \pm} T$, and (3) $R\left(W^{ \pm}\right) \subset \mathscr{R}\left(1-E_{p}\right)$ or $E_{p} W^{ \pm}=0$. Property (2) yields that $W^{ \pm} W^{ \pm *}$ are projections from $\mathcal{H}$ into $\Re\left(W^{ \pm}\right)$. If $\Re\left(W^{+}\right)=\mathscr{R}\left(W^{-}\right)$, then $S$ is unitary. We call $W^{ \pm}$complete iff $W^{ \pm} W^{ \pm *}=1-E_{p}$.

If we replace the $t$-limit in the definition of $W^{ \pm}$by the weaker Abel-limit, then the resulting operators are called stationary wave operators. They may exist even if $W^{ \pm}$do not. However, if $W^{ \pm}$do exist, then the stationary wave operators exist and equal $W^{ \pm}$. It is convenient to define

$$
Z^{ \pm}=\text {s-Abel-lim } e_{t \rightarrow \pm \infty}^{i T t} e^{-i H t} \text {. }
$$

If $W^{ \pm}$and $Z^{ \pm}$exist then $W^{ \pm *}=Z^{ \pm}$. The (stationary) wave operators and scattering matrix can be expressed in terms of the resolvents $R(z)=(H-z)^{-1}$ and $R_{0}(z)=(T-z)^{-1}$ (see [14], [19]), e.g.,

$$
Z^{+}=s-\lim Z^{(\varepsilon)}, \quad Z^{(\varepsilon)}=\frac{\varepsilon}{\pi} \int R_{0}(\lambda-i \varepsilon) R(\lambda+i \varepsilon) d \lambda .
$$

In the stationary case, where the integration over the spectral parameter is involved, it is useful to consider a local version of (III.2). We define for any Borel $\Delta \subset \mathbf{R}:$

$$
Z^{+}(\Delta)=\operatorname{s-lim}_{\varepsilon \rightarrow \pm 0} Z^{(\varepsilon)}(\Delta), \quad Z^{(\varepsilon)}(\Delta)=\frac{\varepsilon}{\pi} \int_{\Delta} R_{0}(\lambda-i \varepsilon) R(\lambda+i \varepsilon) d \lambda .
$$

These operators are the central objects in the stationary theory and $W^{ \pm}$are recovered through $W^{ \pm}(\Delta)=Z^{ \pm}(\Delta)^{*}$ by (see [19])

LEMMA III.1. Let $Z^{ \pm}(\Delta)$ exist for all $\Delta^{\prime}$ 's from a directed sequence $\phi=\{\Delta\}$ of Borel subsets of $\mathbf{R}$ and satisfy $Z^{ \pm}(\Delta)^{*} Z^{ \pm}\left(\Delta^{\prime}\right)=E\left(\Delta \cap \Delta^{\prime}\right)$ for any $\Delta, \Delta^{\prime} \in \phi$. Assume that the Lebesgue measure of $\mathbf{R} \backslash \cup_{\Delta \in \phi} \Delta$ is zero. Then $s-\lim _{\Delta \rightarrow \cup \Delta} Z^{ \pm}(\Delta)$ and $Z^{ \pm}$exist and are equal.

Note that if $W^{ \pm}$are defined in a stationary way, then their isometry does not follow from their existence and has to be proven using additional information about $H$ and $T$. 
Let

$$
\Pi=\int^{\oplus} \Pi_{\lambda} d \lambda, \quad \delta_{e}(H-\lambda)=\frac{1}{\pi} \operatorname{Im} R(\lambda+i \varepsilon), \quad T(z)=V-V R(z) V .
$$

THEOREM III.2. Let there exist a Banach space $X$ with $X \cap \mathcal{H}$ dense in $X$ such that: (i) for any compact interval $\Delta \subset \mathbf{R} \backslash \sigma_{p}(H), \delta_{\varepsilon}(T-\lambda) u(\lambda)$ can be extended to a family of uniformly (in $\varepsilon \in \mathbf{R}^{+}$) bounded operators from $L^{2}(\Delta, X)$ to $L^{2}\left(\Delta, X^{\prime}\right)$, where $X^{\prime}$ is dual to $X$; (ii) $(H-T) R(\cdot+i \varepsilon) f \in L^{2}(\Delta, X)$ and has strong limits in $L^{2}(\Delta, X)$ as $\varepsilon \rightarrow \pm 0$ for any $f$ from a dense set $Y \subset \mathcal{H}$. Then (a) $\sigma_{\text {s.c. }}(H)=\varnothing$, (b) $Z^{ \pm}(\Delta)$ exist (as strong limits), (c) $W^{ \pm}(\Delta) Z^{ \pm}\left(\Delta^{\prime}\right)=E\left(\Delta \cap \Delta^{\prime}\right)$.

Proof. See Appendix II.

COROLlaRY III.3. Under the conditions of Theorem III.2 the global wave operators exist and $Z^{ \pm}=\mathrm{s}-\lim _{\Delta \rightarrow \mathbf{R}} Z^{ \pm}(\Delta)$.

THEOREM III.4. Let there exist a Banach space $X$ with $X \cap \mathcal{H}$ dense in $X$ and such that: $(\alpha) \delta_{e}(T-\lambda)$ can be extended to a family of operators from $X$ to its dual $X^{\prime}$, uniformly bounded in $\varepsilon \in \mathbf{R}^{+}$and $\lambda \in \mathbf{R} ;(\beta)(H-T) R(z), z \in \mathbf{C} \backslash \mathbf{R}$, can be extended to a family of bounded operators on $X$ which have strong boundary values on $\mathbf{R} \backslash \sigma_{p}(H)$.

Then $T_{\lambda} \equiv$ s-lim $_{\varepsilon \downarrow 0} \Pi_{\lambda} T(\lambda+i \varepsilon) \Pi_{\lambda}^{*}$ exists as a measurable $B\left(\mathcal{F}_{\lambda}\right)$-valued function and $S(\lambda)=1_{\lambda}+2 \pi i T_{\lambda}$.

If, in addition, $\delta_{\varepsilon}(T-\lambda)$ is norm continuous as $\varepsilon \downarrow 0$, then $T_{\lambda}$ is strongly continuous in $\lambda \in \mathbf{R} \backslash \sigma_{p}(H)$.

Proof. We begin with

LeMMA III.5. For some Banach space $X$ with $X \cap \mathcal{H}$ dense in $X$, let $\delta_{e}(T-\lambda)$ be bounded from $X$ to $X^{\prime}$ uniformly in $\varepsilon \in \mathbf{R}^{+}$and $\lambda \in \mathbf{R}$. Then $\Pi_{\lambda}$, as a family of operators from $X$ to $\mathcal{H}_{\lambda}$, is uniformly bounded in $\lambda \in \mathbf{R}$. If, in addition, $\delta_{e}(T-\lambda)$ has weakly continuous boundary values as $\varepsilon \downarrow 0$, then $\left\|\Pi_{\lambda} u\right\|_{\mathscr{C}_{\lambda}}$ is continuous in $\lambda$.

Proof. First of all for any $u \in \mathcal{H},\left\|\Pi_{\lambda} u\right\|_{\mathscr{F}} \in L^{2}(\mathbf{R})$. We can write $\left\langle\delta_{e}(T-\lambda) u, u\right\rangle=\int \delta_{e}(s-\lambda)\left\|\Pi_{s} u\right\|_{\mathscr{C}_{s}}^{2} d s$, a Poisson integral of $\left\|\Pi_{s} u\right\|_{\mathscr{C}_{s}}^{2}$. On the other hand, since $\left\langle\delta_{\varepsilon}(T-\lambda) u, u\right\rangle$ is harmonic, positive, and uniformly bounded in $\lambda \in \mathbf{R}$ and $\varepsilon \in \mathbf{R}^{+}$, it is a Poisson integral of a uniformly bounded function, to which it converges as $\varepsilon \downarrow 0$ in the *-weak topology of $L^{\infty}$ [22]. The proof now follows from the uniqueness of the Poisson integral.

LEMMA III.6. If $W^{ \pm}$exist then

$$
S-1=\lim _{\varepsilon \downarrow 0} 2 \pi i \int \delta_{\varepsilon}(T-\lambda) W^{+*} V \delta_{e}(T-\lambda) d \lambda \quad \text { (strongly) }
$$

Proof. Using the definition of $S$ and $W^{ \pm}=\lim _{e \rightarrow \pm 0} W^{(e)}$,

$$
W^{(e)}=1-\int R(\lambda+i \varepsilon) V \delta_{e}\left(H_{0}-\lambda\right) d \lambda,
$$


we obtain

$$
S-1=\lim _{\varepsilon \downarrow 0} W^{+*}\left(W^{(-\varepsilon)}-W^{(e)}\right)
$$

substituting into here the expression for $W^{(e)}$ and taking into account the intertwining property of $W^{+*}$, one finds (III.3).

Now we proceed to the proof of Theorem III.4. The properties of $T_{\lambda}$ follow directly from Lemma III.5 and conditions $(\alpha)$ and $(\beta)$. To obtain $S(\lambda)=\mathbf{1}_{\lambda}+T_{\lambda}$, we replace $W^{+*}$ in (III.3) by $1-\lim _{\varepsilon^{\prime} \downarrow 0} \int \delta_{e^{\prime}}(T-\nu) V R\left(\nu+i \varepsilon^{\prime}\right) d \nu$, take the diagonal $\varepsilon^{\prime}=\varepsilon$ in the limit, apply to the obtained equation $\Pi_{\lambda}$ from the left and $\Pi_{\lambda^{\prime}}^{*}$ from the right and integrate out the variable $\nu$ (to justify this integration one uses conditions $(\alpha)$ and $(\beta))$.

Examples of the spaces $X$.

Definition III.7 (T. KaTO). A closed operator $A$ is relatively smooth with respect to a selfadjoint operator $T$ (or $T$-smooth) iff $D(T) \subset D(A)$ and

$$
\int_{-\infty}^{\infty}\|A R(\lambda \pm i \varepsilon) u\|^{2} d \lambda \leqslant M\|u\|^{2}, \quad M<\infty,
$$

for any $u \in D(T)$ and $\varepsilon>0$.

Denote by $A \mathcal{H}$ the completion of the range of $A$ in the norm $\|x\|_{A \mathcal{K}}=$ $\inf _{h, A h=x}\|h\|_{\mathcal{F C}}$ Note that $A \mathcal{H} \approx \mathcal{H} / \operatorname{Ker} A$. In this notation, $\Sigma A_{i} \mathcal{H C}=j\left(\bigoplus A_{i} \mathcal{H}\right)$, where $j\left(\bigoplus x_{i}\right)=\Sigma x_{i}$.

LEMMA III.8. Let operators $A_{i}$ on $\mathcal{H}$ be $T$-smooth and define $X=\sum A_{i}^{*} \mathcal{H}$. Then $X \cap \mathcal{H}$ is dense in $X$ and $\delta_{\varepsilon}(T-\lambda)$ is bounded from $X$ to $X^{\prime}$ uniformly in $\varepsilon \in \mathbf{R}^{+}$ and $\lambda \in \mathbf{R}$. If, in addition, $A_{i} \delta_{e}(T-\lambda) A_{j}^{*}$ is weakly continuous on $\mathcal{H}$ as $\varepsilon \downarrow 0$ then so is $\delta_{\boldsymbol{e}}(T-\lambda)$ on $X$ to $X^{\prime}$.

Proof. $X \cap \mathcal{H}$ dense in $X$ by the definition of $X$ as the completion of $\Sigma R\left(A_{i}\right)$ in the norm of $X$. Recall now that $A_{i}$ is $T$-smooth if and only if $A_{i} \delta_{e}(T-\lambda) A_{i}^{*}$ is uniformly in $\varepsilon \in \mathbf{R}^{+}$and $\lambda \in \mathbf{R}$ bounded on $\mathcal{H}$. This implies that

$$
\left\|A_{j} \delta_{\varepsilon}(T-\lambda) A_{i}^{*}\right\|^{2} \leqslant\left\|A_{j} \delta_{\varepsilon}(T-\lambda) A_{j}^{*}\right\|\left\|A_{i} \delta_{\varepsilon}(T-\lambda) A_{i}^{*}\right\|
$$

is uniformly bounded on $\mathcal{H}$ for any $i$ and $j$. The latter implies the desired statement.

REMARK III.9. Another proof of the lemma would be to use an equivalent Kato's definition of the relative smooth operator, which implies readily that $\Sigma A_{i}$ is $T$-smooth. Then one uses the equivalence of the norms in $\Sigma A_{i} \mathcal{H}$ and $\left(\Sigma A_{i}\right) \mathcal{H}$.

IV. Scattering theory for abstract Schrödinger operators. In this section we discuss the stationary scattering theory for abstract Schrödinger operators. As a basis for the discussion we take an abstract Hilbert space scattering theory outlined in §III. Below $H=T+\Sigma V_{a}$ is an abstract Schrödinger operator and $W^{ \pm}(\Delta)$ are the local wave operators for $(H, T)$. For the other notation see $\S \S I$ and III. We assume that $\sigma_{\text {ess }}(H) \subset \mathbf{R}$, which holds for single-channel $H$. 
THEOREM IV.1. Assume that $\left|V_{a}\right|^{1 / 2}$ are $T$-smooth and $\left|V_{a}\right|^{1 / 2} R(z)\left|V_{b}\right|^{1 / 2}$, for any $a, b \in \mathbb{Q}$, is a family of bounded operators on $\mathcal{H}$ strongly continuous as $z \rightarrow$ $\mathbf{R} \backslash \sigma_{p}(H)$. Then $(1) \sigma_{\text {s.c. }}(H)=\varnothing,(2) Z^{ \pm}(\Delta)$ exist for all compact $\Delta \subset \mathbf{R} \backslash \sigma_{p}(H)$ as strong limits, (3) $W^{ \pm}(\Delta) Z^{ \pm}\left(\Delta^{\prime}\right)=E\left(\Delta \cap \Delta^{\prime}\right)$, and (4) $T_{\lambda} \equiv \lim _{e \downarrow 0} \Pi_{\lambda} T(\lambda+i \varepsilon) \Pi_{\lambda}^{*}$ exists as a measurable $B\left(\mathcal{H}_{\lambda}\right)$-valued function uniformly bounded in $\lambda \in \mathbf{R}$ and $S(\lambda)=\mathbf{1}_{\lambda}+T_{\lambda}$.

Proof. The statement follows from Theorems III.2 and III.4 with the Banach space

$$
Y=X=\sum\left|V_{a}\right|^{1 / 2} \mathcal{H}
$$

and Lemma III.5.

REMARK IV.2. One can show also that under the conditions of Theorem IV.1, $W^{ \pm}(\Delta)$ exist as strong (stationary) limits and satisfy $Z^{ \pm}(\Delta) W^{ \pm}\left(\Delta^{\prime}\right)=E_{0}\left(\Delta \cap \Delta^{\prime}\right)$.

Our next task is to reduce the condition of Theorem IV.1 on $R(z)$ to restrictions on the basic operators $T$ and $V_{a}$. The following statement is the first step in this program. Define

$$
\begin{gathered}
V^{1 / 2}=(\operatorname{sign} V)|V|^{1 / 2}, \quad H_{a}(g)=T+\sum_{b \subset a} g_{b} V_{b}, \quad R_{a}(z, g)=\left(H_{a}(g)-z\right)^{-1}, \\
G=\left\{g: \sigma_{p}\left(H_{a}(g)\right)=\varnothing \text { for all } a \neq a_{\max }\right\} .
\end{gathered}
$$

THEOREM IV.3. Let the following conditions be satisfied:

(i) For all $b, c$ and $a$, with $b, c \subset a$ if $a \neq a_{\max }$, the operators $\left|V_{b}\right|^{1 / 2} R_{a}(z, g)\left|V_{c}\right|^{1 / 2}$ are bounded on $\mathcal{H}$, analytic in $z \in \mathrm{C} \backslash \sigma(T)$ and $g \in G$ and strongly continuous as $z \rightarrow \pm 0$ uniformly in $g \in G$.

(ii) There is an integer $s>0$ such that the product of $s$ operator-functions of the form

$$
\prod_{i=1}^{k}\left[V_{b_{i}}^{1 / 2} R_{0}(z)\left|V_{b_{i+1}}\right|^{1 / 2}\right], \quad \cup b_{i}=a_{\max },
$$

is compact on $\mathcal{H}$ together with its boundary values on $\sigma(T)$.

(iii) $(H(g)-\lambda) \psi=0, \quad \psi \in R_{0}(\lambda \pm i 0) \Sigma\left|V_{a}\right|^{1 / 2} \mathcal{H} \Rightarrow$ either $\psi=0$ or $\lambda \in$ $\sigma_{p}(H(g))$.

Then for any $b, c \in \mathbb{Q}$ and $g \in G,\left|V_{b}\right|^{1 / 2} R(z, g)\left|V_{c}\right|^{1 / 2}$ are bounded on $\mathcal{H}$, analytic in $z \in C \backslash \sigma(T) \cup \sigma_{p}(H(g))$ and $g \in G$, strongly continuous as $z \rightarrow$ $\mathbf{R} \backslash \sigma_{p}(H(g))$ uniformly in $g \in G$.

REMARK. For the sake of explicitness we write condition (iii) here and analogous conditions below in a somewhat formal way. To make them precise one should consider $(H-\lambda) R_{0}(\lambda \pm i 0) \equiv 1+\sum V_{a} R_{0}(\lambda \pm i 0)$ on $\Sigma\left|V_{a}\right|^{1 / 2} \mathcal{H}$ instead of $H-\lambda$ on $R_{0}(\lambda \pm i 0) \Sigma\left|V_{a}\right|^{1 / 2} \mathcal{K}$. The former family is well defined for all $\lambda$ (and strongly continuous in $\lambda$ ) by virtue of (i).

Proof. Consider the operator $L(z, g)=A(z, g)-1$. Writing $L(z, g)=$ $\Sigma_{f}\left|V_{f}\right|^{1 / 2} L_{f}(z, g)$ and defining $L_{f g}=L_{f}\left|V_{g}\right|^{1 / 2}$ and $F_{f g}=V_{f}^{1 / 2} F\left|V_{g}\right|^{1 / 2}$, we obtain

$$
V_{c}^{1 / 2} R\left|V_{g}\right|^{1 / 2}+\sum_{f} V_{c}^{1 / 2} R\left|V_{f}\right|^{1 / 2} L_{f g}=F_{c g}
$$


The operators $L_{c d}$ and $F_{c d}$ are linear combinations of monomials of the form

$$
\stackrel{\stackrel{k}{\longrightarrow}}{\prod_{i=1}}\left[V_{f_{i}}^{1 / 2} R_{b_{i}}\left|V_{f_{i+1}}\right|^{1 / 2}\right], \quad b_{i} \neq a_{\max },
$$

$f_{1}=c, f_{k+1}=d$ with the conditions $\cup f_{i}=a_{\max }$ and $f_{i} \neq a_{\max }$, respectively.

We transform (IV.3) so that each factor satisfies $f_{i}, f_{i+1} \subseteq b_{i}$ if $a \neq a_{\max }$. To this end we use the equations $R_{b}=F_{b}-R_{b} L_{b}$ and $R_{b}=F_{b}^{\prime}-L_{b}^{\prime} R_{b}$, where $F_{b}^{\prime}(z)=$ $F_{b}(\bar{z})^{*}$ and $L_{b}^{\prime}(z)=L_{b}(\bar{z})^{*}$, to surround each $R_{b}$ in (IV.3) with $V_{f}^{1 / 2}$, next on its left, and $\left|V_{g}\right|^{1 / 2}$, next on its right, satisfying $f, g \subseteq b$.

Proposition IV.4. The operators $L_{f d}(z, g)$ and $F_{f d}(z, g)$ are bounded on $\mathcal{H}$, analytic in $z \in C \backslash \sigma(T)$ and $g \in G$ and strongly continuous in $z$, uniformly in $g \in G$, as $z$ approaches $\sigma(T)$.

Proof. The statement follows from assumption (i) using the transformation described in the paragraph preceding the proposition.

Proposition IV.5. The matrix $\left[L_{f d}(z, g)\right]^{s}, g \in G$, is compact for all $z \in C \backslash \mathbf{R} u p$ to the real axis.

Proof. Since the matrix is analytic in $g \in G$, it suffices to prove the proposition for a neighborhood of $g=0$. It follows from condition (i) that the series (II.5) with $V_{c} \rightarrow g_{c} V_{c}$ converges in the norm for all $z \in \mathbf{C} \backslash \mathbf{R}$ up to the real axis, as long as $g$ is confined to a neighborhood of 0 . Substituting such series for $R_{b}$ 's in (IV.3) we conclude that $L_{f d}(z, g)$ for $g$ in a neighborhood $V$ of zero is a norm convergent series of terms of the form

$$
g^{k} \prod_{\cup f_{i}=a_{\max }}\left[V_{f_{i}}^{1 / 2} R_{0}\left|V_{f_{i+1}}\right|^{1 / 2}\right] .
$$

Then the matrix $\left[L_{f d}(z)\right]^{s}$ for $g \in V$ is a norm convergent series of terms, each of which is, by virtue of condition (ii), a compact operator on $\bigoplus \mathcal{H C}$ for all $z \in \mathbf{C} \backslash \mathbf{R}$ up to the real axis. By the theorem on the closedness of the set of compact operators in the uniform topology, $\left[L_{f d}(z, g)\right]^{s}$ is compact as well (for $g \in V$ ).

PROPOSITION IV.6. The following two statements are equivalent:

$(\alpha)-1 \in \sigma\left(\left[L_{f d}(z, g)\right]\right)$.

( $\beta) z \in \sigma_{p}(H(g))$.

Proof. If $f+\left[L_{c d}(z, g)\right] f=0, f=\bigoplus f_{b} \in \bigoplus \mathcal{H}$, then $\chi=\Sigma\left|V_{a}\right|^{1 / 2} f_{b}$ satisfies $A(z, g) \chi=0$ and, therefore, by virtue of (II.3), $\varphi=F(z, g) \chi$ obeys formally (in the weak sense) $(H-z) \varphi=0$. If $z \notin \sigma(T)=\bigcup \sigma\left(H_{a}(g)\right)$, then, obviously, $\varphi \in D(T)$ $=D(H(g))$ and therefore $\varphi$ is an eigenvector of $H(g)$ corresponding to the eigenvalue $z$. Otherwise we use condition (3).

Propositions IV.4-IV.6 imply the statement of Theorem IV.3.

There are two ways to prove condition (iii), starting from assumptions on $T$ and $V_{a}$ (the argument $g$ is suppressed in what follows). The first is based on the observation [19] that (i) and (ii) imply, for selfadjoint $H$, that $\lim _{e \downarrow 0}\left(\delta_{e}(T-\lambda) \varphi, \varphi\right)$ $=0$ for $\varphi \in R_{0}(\lambda \pm i 0) \Sigma\left|V_{a}\right|^{1 / 2 \mathcal{H}}$, a weak solution of $(H-\lambda) \varphi=0$. Then by 
strengthening somewhat conditions (i) and (ii) one can obtain a necessary smoothness for $\varphi$ to guarantee that $\varphi \in D(H)$.

In the second way condition (iii) is replaced by the following two conditions:

(iii $\left.{ }_{1}\right)$ There is a unitary representation $\rho \rightarrow U(\rho)$ of $\overline{\mathbf{R}}^{+}$on $\mathcal{H}$ such that $U(\rho) T U(\rho)^{-1}$ and $U(\rho) V_{a} U(\rho)^{-1}, a \in \mathcal{Q}$, have analytic continuations $T(\zeta)$ and $V_{a}(\zeta)$ from $\mathbf{R}$ to $\theta \subset \mathbf{C}, \Theta \cap \overline{\mathbf{R}}^{+} \neq \varnothing$, the former on $D(T)$ and the latter as an operator from $D(T)$ to $\mathcal{H}$.

(iii $)_{2}(H-\lambda) \varphi=0, \lambda \in \sigma(T) \cap \sigma(T(\zeta)), \operatorname{Im} \zeta \neq 0, \varphi \in R_{0}(\lambda \pm i 0) \Sigma V_{a} \mathcal{H} \Leftrightarrow$ either $\varphi=0$ or $\lambda \in \sigma_{p}(H)$.

REMARK IV.7. The advantage of new conditions ( $\left.\mathrm{iii}_{1}\right)$ and $\left(\mathrm{iii}_{2}\right)$ lies in the fact that the set $\sigma(T) \cap \sigma(T(\zeta))$, $\operatorname{Im} \zeta \neq 0$, usually is small and (iii ${ }_{2}$ ) can be easily proven.

EXAMPLE IV.8. Let $T$ be unitary equivalent to a multiplication operator $\hat{T}$ of the form

$$
\hat{T} \hat{f}=\bigoplus\left(\lambda f_{i}(\lambda)\right), \quad \hat{f}=\bigoplus f_{i} \in \hat{\mathcal{H}} \equiv \bigoplus L^{2}\left(\left[E_{i}, \infty\right), \mathcal{H}_{i}\right),
$$

where $E_{i} \in \mathbf{R}$ and $\mathcal{H}_{i}$ are Hilbert spaces, and let $U$ be the corresponding unitary operator from $\mathcal{H}$ to $\mathcal{H}$. We can define $U(\rho)$ as

$$
U U(\rho) f=\rho^{1 / 2}\left[\bigoplus(U f)_{i}\left(\rho\left(\lambda-E_{i}\right)+E_{i}\right)\right] .
$$

Then $\sigma(T) \cap \sigma(T(\zeta))=\left\{E_{i}\right\}$ for $\operatorname{Im} \zeta \neq 0$.

Proposition IV.9. (iii 1 ) and ( iii $_{2}$ ) imply (iii).

The proof follows readily from the following

Proposition IV.10. Let $\lambda \in \sigma(T) \backslash \sigma(T(\zeta)), \operatorname{Im} \zeta \neq 0$, and $\varphi \in$ $R_{0}(\lambda \pm i 0) \Sigma\left|V_{a}\right|^{1 / 2} \mathcal{H}$ be a weak solution to $(H-\lambda) \varphi=0$. Then either $\varphi=0$ or $\lambda \in \sigma_{p}(H)$.

Proof. The following two conditions are equivalent by the definition of $L_{c d}$ (see the proof of Proposition IV.6):

$(H-\lambda) \varphi=0$ has a nontrivial weak solution from $R_{0}(\lambda \pm i 0) \Sigma\left|V_{a}\right|^{1 / 2} \mathcal{H}$ and $-1 \in \sigma\left(\left[L_{c d}(\lambda \pm i 0)\right]\right)$.

We define $L_{c d}(z, \zeta)$ for $H(\zeta)=T(\zeta)+\Sigma V_{a}(\zeta) . L_{c d}$ is analytic in $\zeta$ as long as $z \notin \sigma_{\text {ess }}(H(\zeta))$. Therefore by Lemma AI.3,

$$
-1 \in \sigma\left(\left[L_{c d}(\lambda \pm i 0)\right]\right) \Leftrightarrow-1 \in \sigma\left(\left[L_{c d}(\lambda, \zeta)\right]\right), \quad \operatorname{Im} \zeta \neq 0 .
$$

Furthermore, as in Proposition IV.6,

$$
-1 \in \sigma\left(\left[L_{c d}(\lambda, \zeta)\right]\right) \Leftrightarrow \lambda \in \sigma_{d}(H(\zeta)) \text { for } \lambda \notin \sigma_{\text {ess }}(H(\zeta))
$$

The following lemma, which is a simple extension of one of the Balslev-Combes theorems [15] (the proof is exactly the same), completes the chain.

LEMMA IV.11. Let $H(\zeta)$ be as defined above. Then

$$
\sigma_{d}(H(\zeta)) \cap \mathbf{R}=\sigma_{p}(H) \cap \mathbf{R} .
$$


Proof. It suffices to prove that $\sigma_{d}(H(\zeta)) \cap \mathbf{R} \subset \sigma_{p}(H)$. The other direction follows if one exchanges roles of $H$ and $H(\zeta)$ (say, define $H_{\text {new }}=H(\zeta)$ ). Let $F$ and $\phi$ be $U(\rho)$-analytic vectors. We have

$$
\langle\phi, R(z) F\rangle=\langle\phi(\zeta), R(z, \zeta) F(\zeta)\rangle,
$$

where $\phi(\zeta)$ is an analytic continuation of $U(\rho) \phi$. This implies the (ᄃ)-inclusion.

The proof of Propositions IV.10 and IV.9 is completed.

V. Abstract many-body systems. The task of this section is to derive the properties of $H$ required in Theorem IV.1 essentially from restrictions on the basic operators $T$ and $V_{a}, a \in \mathbb{Q}$. In the previous section we have reduced the desired statement about $H$ to restrictions on $R_{a}, a \in \mathbb{Q}$, and on $T$ and $V_{a}, a \in \mathbb{Q}$. To continue this process so as to end up with conditions only on $T$ and $V_{a}$, we have to give some additional structure to the space $\mathcal{H}$ and operator $H$.

Definition V.1. Let $\mathbb{Q}$ be a finite lattice. With each pair $(b, a) \in \mathbb{Q} \times \mathbb{Q}$ such that $b \subset a$, we associate a Hilbert space $\mathcal{F}_{b}^{a}$ and a positive, absolutely continuous operator $T_{b}^{a}$ on $\mathcal{F F}_{b}^{a}$. We denote $\mathcal{H}_{b}=\mathcal{H}_{b}^{a}$ and $T_{b}=T_{b}^{a}$ if $a=a_{\max }$, and $\mathcal{H C}^{a}=\mathcal{F C}_{b}^{a}$ and $T^{a}=T_{b}^{a}$ if $b=a_{\min }$ (we omit an index if it is $a_{\max }$ or $a_{\min }$ ). Moreover, to every $a$ we assign an operator $V^{a}$ on $\mathcal{F}^{a}$ and denote $V_{b}^{a}=V^{b} \otimes \mathbf{1}_{b}^{a}$. We call a collection $\left\{\mathcal{F}_{b}^{a}, T_{b}^{a}, V^{a}, b \subset a \in \mathbb{Q}\right\}$ (or simply $\left\{T_{b}^{a}, V^{a}\right\}$ ) an abstract many-body system (AMBS) if and only if the following conditions are satisfied:

$$
\begin{aligned}
& \mathcal{F}_{c}^{a}=\mathcal{H}_{c}^{b} \otimes \mathcal{H}_{b}^{a} \quad \text { for } c \subseteq b \subseteq a, \\
& T_{c}^{a}=T_{c}^{b} \otimes \mathbf{1}_{b}^{a}+\mathbf{1}_{c}^{b} \otimes T_{b}^{a} \quad \text { for } c \subseteq b \subseteq a
\end{aligned}
$$

and

(B) for any $a, V^{a}$ is $T^{a}$-compact.

Definition V.2. The operator $H=T+\Sigma V_{a}$, defined on $D(T)$ by virtue of (B), is called the Schrödinger operator for $\left\{T_{b}^{a}, V^{a}\right\}$. If $V^{a}$ are symmetric, then $H$ is selfadjoint.

The following operators play an important role in the many-body problem:

$$
H_{a}=T+\sum_{b \subseteq a} V_{b} \text { and } H^{a}=T^{a}+\sum_{b \subseteq a} V_{b}^{a} .
$$

Note that

$$
H_{a}=H^{a} \otimes 1_{a}+1^{a} \otimes T_{a}
$$

Spectral theory. For notational convenience we set $\sigma_{p}\left(H^{a}\right)=\{0\}$ for $a=a_{\min }$.

Proposition V.3. Let $\left\{T_{b}^{a}, V^{a}\right\}$ be an AMBS. Then $\sigma\left(T^{a}\right) \subset \sigma_{\text {ess }}\left(H^{a}\right)$ for each $a \in \mathbb{Q}$.

Proof. Since $V^{b}$ is $T^{b}$-compact, $V^{b} \exp \left(-i T^{b} t\right) \stackrel{s}{\rightarrow} 0$ on $D\left(T^{b}\right)$ to $\mathcal{H}^{b}$ as $|t| \rightarrow \infty$, and therefore $V_{b}^{a} \exp \left(-i T^{a} t\right) \stackrel{s}{\rightarrow} 0$ on $D\left(T^{a}\right)$ to $\mathcal{F}^{a}$ as $|t| \rightarrow \infty$ for all $b \subset a$. Applying Proposition I.1 we complete the proof. 
THEOREM V.4. Suppose an abstract many-body system $\left\{T_{b}^{a}, V^{a}\right\}$ satisfies condition (A) of Theorem I.2 for each $H^{a}, a \in \mathcal{Q}$. Then

$$
\sigma\left(T^{a}\right) \subseteq \sigma_{\mathrm{ess}}\left(H^{a}\right) \subseteq \bigcup_{b \subset a}\left[\sigma_{p}\left(H^{b}\right)+\sigma\left(T_{b}^{a}\right)\right],
$$

where the union extends only over the b's with $\sigma_{p}\left(H^{b}\right) \neq \varnothing$.

Proof. The inclusion $\sigma\left(T^{a}\right) \subset \sigma_{\text {ess }}\left(H^{a}\right)$ follows from Proposition V.3. The other direction follows from Theorem I.2, applied to each $H^{a}$, and (V.2).

REMARK V.5. If we assume that for each eigenvalue $\lambda \in \sigma_{p}\left(H^{b}\right)$ with the projection $P_{\lambda}^{b}$ and for each $c \in \mathbb{Q}$, the operator $V_{c}^{d}\left(P_{\lambda}^{b} \otimes \mathbf{1}_{b}^{d}\right)$, where $d=b \cup c$, is $\left(1^{b} \otimes T_{b}^{d}\right)$-compact, then $\cup_{b \subset a}\left[\sigma_{p}\left(H^{b}\right)+\sigma\left(T_{b}^{a}\right)\right] \subset \sigma_{\text {ess }}\left(H^{a}\right)$. The proof of this fact goes exactly as that of Proposition V.3. Combining this statement with Theorem V.4 we obtain that under the joint conditions,

$$
\sigma_{\text {ess }}\left(H^{a}\right)=\bigcup_{b \subset a}\left[\sigma_{p}\left(H^{b}\right)+\sigma\left(T_{b}^{a}\right)\right] .
$$

CoROllaRY V.6. If $\sigma_{d}\left(H^{a}\right)=\varnothing$ for all $a \in \mathbb{Q} \backslash\left\{a_{\max }\right\}$, then $\sigma_{\text {ess }}\left(H^{a}\right)=\sigma\left(T^{a}\right)$.

Definition V.7. An abstract many-body system with $\sigma_{p}\left(H^{a}\right)=\varnothing$ for all $a \in$ $\mathbb{Q} \backslash\left\{a_{\max }\right\}$ will be called the abstract, single channel, many-body system.

We say that an abstract many-body system, $\left\{T_{b}^{a}, V^{a}\right\}$, is strongly single channel iff $\left\{T_{b}^{a}, g_{a} V^{a}\right\}$ are single channel for all $\left\{g_{a}\right\}$ in a neighborhood of one (roughly speaking, if it remains single channel under small perturbations).

Resolvent on the continuous spectrum and scattering theory. Denote by $G^{a}$ a connected subset of $\left\{g, \sigma_{p}\left(H^{b}(g)\right)=\varnothing \forall b \subset a\right\}$, containing the origin $g=0$. Recall that $H^{b}(g)=T^{b}+\Sigma_{c \subset b} g_{c} V_{c}^{b}$. Let $E_{p}^{a}(g)$ be the projection onto the subspace of the point spectrum of $H^{a}(g)$.

TheOREM V.8. Assume that an abstract, many-body system $\left\{T_{b}^{a}, V^{b}\right\}$ satisfies for all $a \in \mathbb{Q}:$

(i) For all $c, d \subseteq a$, the operators $\left|V_{c}^{a}\right|^{1 / 2}\left(T^{a}-z\right)^{-1}\left|V_{d}^{a}\right|^{1 / 2}$ are bounded on $\mathcal{H}^{a}$ and strongly continuous as $\operatorname{Im} z \rightarrow \pm 0$.

(ii) There is an integer $s>0$ such that the product of $s$ operators of the form

$$
\prod_{i=1}^{\stackrel{k}{\rightarrow}}\left[\left|V_{b_{i}}^{a}\right|^{1 / 2}\left(T^{a}-z\right)^{-1}\left|V_{b_{i+1}}^{a}\right|^{1 / 2}\right], \quad \bigcup_{1}^{k+1} b_{i}=a,
$$

is compact together with its boundary values on $\sigma\left(T^{a}\right)$.

Moreover, assume

(iii) $H^{a}(g) \varphi=\lambda \varphi, \varphi \in R_{0}(\lambda \pm i 0) \Sigma_{b \subseteq a}\left|V_{b}^{a}\right|^{1 / 2} \mathcal{F}^{a} \Rightarrow$ either $\varphi=0$ or $\lambda \in$ $\sigma_{p}\left(H^{a}(g)\right)$.

Then $\left|V_{c}^{a}\right|^{1 / 2} R^{a}(z, g)\left|V_{d}^{a}\right|^{1 / 2}$ for each $c, d \subseteq a$ is a family of bounded operators on $\mathcal{H}^{a}$, analytic in $z \in \mathbf{C} \backslash\left[\sigma\left(T^{a}\right) \cup \sigma_{p}\left(H^{a}(g)\right)\right]$ and $g \in G^{a}$ strongly continuous in $z$ uniformly in $g \in G^{a}$ up to the boundary $\sigma\left(T^{a}\right) \backslash \sigma_{p}(H(g))$.

Proof. We conduct the proof by induction on $a \in \mathcal{Q}$. For $a=a_{\min }$ we do not have $H^{a}$. Let the statement be true for all $b, b \subset a$, and prove it for $a$. In the sequel 
we suppress the superindex $a$. In order to apply Theorem IV.3 to $H$ we have to obtain the required information about $R_{b}(z), b \subset a$, on $\mathcal{H}$. To this end we use

LemMA V.9. The operators $\left|V_{c}\right|^{1 / 2} R_{b}(z, g)\left|V_{d}\right|^{1 / 2}, c, d \subseteq b \subset a$, considered on $\mathcal{F C}^{a}$, are bounded analytic in $z \in C \backslash \sigma(T)$ and $g \in G$ and strongly continuous in $z \in$ $C \backslash \mathbf{R}$ uniformly in $g \in G$ up to the real axis.

PRoof. Let $S_{b}=\mathbf{1}^{b} \otimes s_{b}$, where $s_{b}$ is a unitary operator from $\mathcal{H}_{b}$ to the direct integral $\int^{\oplus} \mathcal{F}_{\lambda} d \lambda$ with respect to $T_{b}:\left(s_{b} T_{b}^{a} u\right)(\lambda)=\lambda\left(s_{b} u\right)(\lambda)$. Then $(g$ is omitted)

$$
S_{b}\left|V_{c}\right|^{1 / 2} R_{b}(z)\left|V_{d}\right|^{1 / 2} u=\left|V_{c}\right|^{1 / 2} R^{b}(z-\lambda)\left|V_{d}\right|^{1 / 2}\left(S_{b} u\right)(\lambda) \text {. }
$$

This equation together with the induction statement about $\left|V_{c}^{b}\right|^{1 / 2} R^{b}(z)\left|V_{d}^{b}\right|^{1 / 2}$ implies the lemma.

This completes the proof of Theorem V.8.

ReMARK V.10. For a discussion of condition (iii) see the part of §IV following the proof of Proposition IV.6.

Combining Theorems IV.1 and V.8 we obtain

THEOREM V.11. Assume that a single channel, AMBS satisfies the conditions of Theorem V.8. Then the statement of Theorem IV.1 holds for $H=T+\Sigma V_{a}$.

RemarK V.12. If we consider instead of $H$ the family $H(g)$, then the operators $Z^{ \pm}, W^{ \pm}, S$ and $S(\lambda)$ are analytic in $g \in G$ (definitions of these operators for complex $g$ or nonsymmetric $V^{b}$ 's are given in $\S \mathrm{IX}$ ).

VI. Single channel many-body systems. In this section we describe spectral properties of the Schrödinger operators for many-body systems and establish fundamental properties of the wave operators and scattering matrices associated with these operators. We begin with definitions.

Hamiltonian. Consider a system of $N$ particles in $\mathbf{R}^{\nu}$ with masses $m_{i}$ and interacting via pair potentials $V_{l}\left(x^{l}\right)$. Here $l$ labels pairs of indices and $x^{l}=x_{i}-x_{j}$ for $l=(i j)$. The configuration space of the system in the center-of-mass (CM) frame is defined as $R=\left\{x \in \mathbf{R}^{\nu N}, \Sigma m_{i} x_{i}=0\right\}$ with the inner product $(x, \tilde{x})=$ $\sum m_{i} x_{i} \cdot \tilde{x}_{i}$. Denote by $V^{l}$ and $V_{l}$ the multiplication operators on $L^{2}\left(\mathbf{R}^{\nu}\right)$ and $L^{2}(R)$ by the (real-valued) functions $V_{l}(y)$ and $V_{l}\left(x^{l}\right)$, respectively.

We assume that $V^{l}$ are $\Delta$-compact, i.e. compact as operators from the Sobolev space $H_{2}\left(\mathbf{R}^{\nu}\right)$ to $L^{2}\left(\mathbf{R}^{\nu}\right)$. Then the operator

$$
H=H_{0}+\sum V_{l}, \quad H_{0}=-\frac{1}{2}\left(\text { Laplacian on } L^{2}(R)\right)
$$

is defined on $L^{2}(R)$ and is selfadjoint there.

Partitions. Let $a=\left\{C_{i}\right\}$ be a decompostion of the set $\{1, \ldots, N\}$ into nonempty, disjoint subsets $C_{i}$, called clusters. Denote by $\mathbb{Q}$ the set of all such dr compositions. $Q$ can be given the structure of a lattice; namely, if $b$ is a partition obtained by breaking up certain subsystems of $a$, write $b \subset a$. The smallest partition containing two partitions $a$ and $b$ will be denoted by $a \cup b$, i.e., $a \cup b=$ $\sup (a, b)$. The largest partition contained in both $a$ and $b$ will be denoted by $a \cap b: a \cap b=\inf (a, b)$. 
A pair $l$ will be identified with the decomposition on $N-1$ clusters, one of which is the pair $l$ itself and the others are free particles. Therefore, in the $N$-body case $H_{a}=H_{0}+\Sigma_{l<a} V_{l}$.

Many-body structure. The spaces $\mathcal{H}_{b}^{a}$ are constructed as follows. Define the configuration space of a system of $N$ particles with the centers-of-mass of subsystems $C_{i} \in a$ fixed:

$$
R^{a}=\left\{x \in R, \sum_{j \in C_{i}} m_{j} x_{j}=0 \forall C_{i} \in a\right\}
$$

and the configuration space of the relative motion of the centers-of-mass of the clusters $C_{i} \in a$ :

$$
R_{a}=\left\{x \in R, x_{i}=x_{j} \text { if } i \text { and } j \text { belong to the same } C_{k} \in a\right\} .
$$

Then $R^{a} \perp R_{a}$ and

$$
R^{a} \oplus R_{a}=R, \quad L^{2}\left(R^{a}\right) \otimes L^{2}\left(R_{a}\right)=L^{2}(R) .
$$

Note that $R^{b} \subset R^{a}$ and $R_{a} \subset R_{b}$ for $b \subset a$, so we can define $R_{b}^{a}=R^{a} \ominus R^{b}=R_{b} \ominus$ $R_{a}$. Then $R_{c}^{b} \oplus R_{b}^{a}=R_{c}^{a}$ and $\mathcal{H}_{b}^{a}=L^{2}\left(R_{b}^{a}\right)$ satisfies (V.1). Moreover, $R_{b}=R_{b}^{a}$ for $a=a_{\max }$ and $R^{a}=R_{b}^{a}$ for $b=a_{\text {min }}$.

The operator $T_{b}^{a}$ on $\mathcal{H}_{b}^{a}$ is defined as the selfadjoint extension of $-\frac{1}{2} \Delta_{b}^{a}$, where $\Delta_{b}^{a}$ is the Laplacian on $R_{b}^{a}$. So $\left\{T_{b}^{a}, V^{\prime}\right\}$ is a many-body system in the sense of Definition V.1. it is single channel iff $\sigma_{p}\left(H^{a}\right)=\varnothing$, where $H^{a}=T^{a}+\sum_{l \subseteq a} V_{l}$ on $L^{2}\left(R^{a}\right)$, for all $a \neq a_{\min }, a_{\max }$. Moreover, it follows from Lemmas VI.6a and VI.6b below that a single channel, short range (i.e., $V_{l} \in L^{p} \cap L^{q}\left(\mathbf{R}^{\nu}\right), p>\frac{1}{2} \nu>q$ ) system is strongly single channel iff for any $l, H^{l} \psi=0$ has no nontrivial non- $L^{2}$ solution in $\left(T^{l}\right)^{-1}\left|V^{l}\right|^{1 / 2} L^{2}\left(R^{l}\right)$ (notice that $R^{l}=\mathbf{R}^{\nu}$ and $T^{l}=-\frac{1}{2} \Delta$ on $L^{2}\left(\mathbf{R}^{\nu}\right)$ ). This is an implicit condition on $V^{l}$ which fails rarely (if $V^{l}$ fails to satisfy the condition then $g_{l} V^{\prime}, 0<\left|g_{l}\right|<\varepsilon$, for some $\varepsilon>0$, obeys it).

$X$-operators. Let

$$
X \in C^{\infty}\left(\mathbf{R}^{\nu}\right), \quad X(y)=0 \text { for }|y| \leqslant 1 \text { and } X(y)=1 \text { for }|y| \geqslant 2 .
$$

We define the multiplication operators $X_{n}^{l}$ on $L^{2}\left(R^{l}\right)$ by the functions $X\left(n^{-1} x^{l}\right)$ and the operators $\bar{X}_{n}^{l}=1-X_{n}^{l}$, approximations of identities on $L^{2}\left(R^{l}\right)$. Therefore, since $V^{l}\left(T^{l}-z\right)^{-1}$ is compact, $\bar{X}_{n}^{l} V^{l}\left(T^{l}-z\right)^{-1} \rightarrow 0(n \rightarrow 0)$. Moreover, we compute

$$
\left[T^{l}, X_{n}^{\prime}\right]=n^{-2} F_{n}^{l}+n^{-1} G_{n}^{l} \cdot \nabla^{\prime},
$$

where $\mu_{l}$ is the reduced mass for the pair $l, F_{n}^{l}$ is the multiplication operator by the function $\left(2 \mu_{l}\right)^{-1}(\Delta X)\left(n^{-1} x^{l}\right)$, and the $i$ th component of $G_{n}^{l}$ is the multiplication operator with $\mu_{l}^{-1}\left(\nabla_{i} X\right)\left(n^{-1} x^{l}\right)$. Therefore $\left[T^{l}, X_{n}^{l}\right]\left(T^{l}-z\right)^{-1} \rightarrow 0$ as $n \rightarrow \infty$. We define $X_{l}^{(n)}=X_{n}^{l} \otimes 1_{l}$. Then

$$
\prod_{\cup l=\{(1 \ldots n)\}} \bar{X} l^{(n)} \in C_{0}^{\infty}(R) .
$$

Thus $X_{l}^{(n)}$ satisfy all requirements of Lemma I.3. 
Potentials. Below we describe two classes of pair potentials which satisfy abstract conditions of the previous sections. Proposition VI.1 is given without a proof and Propositions VI.3 and VI.4 are proven in §§VII and VIII, respectively.

Proposition VI.1. The potentials of the class $L^{p}\left(\mathbf{R}^{\nu}\right)+\left(L^{\infty}\left(\mathbf{R}^{\nu}\right)\right)_{e}$, where $p>$ $\max (\nu / 2,2)$ if $\nu \neq 4$ and $p>2$ if $\nu=4$, and subindex $\varepsilon$ indicates that the $L^{\infty}$. component can be taken arbitrary small, are $\Delta$-compact.

Proposition VI.2. The potentials of the class $L^{p} \cap L^{q}\left(\mathbf{R}^{\nu}\right), p>\nu>q$, are $\Delta$-smooth.

Actually, a stronger statement holds for the class above:

Proposition VI.3. Let $U$ and $W$ be the multiplication operators by functions $\varphi\left(x^{l}\right)$ and $\psi\left(x^{s}\right)$, where $\varphi, \psi \in L^{p} \cap L^{q}\left(\mathbf{R}^{\nu}\right), p>\nu>q$ and $l$ and $s$ are arbitrary pairs of indices. The family $W\left(-\Delta^{a}-z\right)^{-1} U$ is bounded on $L^{2}\left(R^{a}\right)$, analytic in $z \in \mathbf{C} \backslash \overline{\mathbf{R}}^{+}$, has strong boundary values on $\overline{\mathbf{R}}^{+}$and is bounded in norm as $\left\|W\left(-\Delta^{a}-z\right)^{-1} U\right\|<$

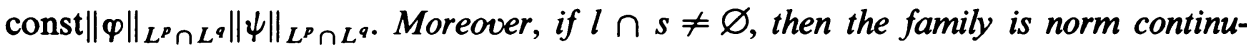
ous as $\operatorname{Im} z \rightarrow \pm 0$.

Proposition VI.4. Let $U_{l}$ and $W_{l}$ be the multiplication operators by functions $\varphi_{I}\left(x^{l}\right)$ and $\psi_{l}\left(x^{l}\right)$, respectively, where $\varphi, \psi \in L^{p} \cap L^{q}\left(R^{\nu}\right), p>\nu>q$. Then a product of three operator-functions $\mathbf{C} \backslash \overline{\mathbf{R}}^{+} \rightarrow B\left(L^{2}\left(R^{a}\right)\right)$ of the form $\Pi\left[W_{l_{i}}\left(-\Delta^{a}-z\right)^{-1} U_{l_{i+1}}\right], \cup l_{i}=a$, has norm-continuous boundary values on $\overline{\mathbf{R}}^{+}$. These boundary values are compact.

The following class of potentials gives rise to single channel systems: $V_{l}$ can be written as a sum of repulsive (i.e. $\partial V_{l} / \partial\left|x^{l}\right| \leqslant 0$ for $x^{l} \neq 0$ [34], [15]) and $|x|^{-2}(1+|x|)^{-1-\varepsilon} L^{\infty}\left(\mathbf{R}^{\nu}\right)$-small Kato potentials. To demonstrate this we use the results of R. Lavine [30], [31], [15] and a modified Iorio-O'Carroll technique [9], [15]: if $V_{l}=V_{l}^{R}+V_{l}^{S}$ in the obvious notation, then it suffices to show that the operators $U R_{1}(\lambda+i 0) W$, where $U$ and $W$ obey $|F(x)| \leqslant C|x|^{-1}(1+|x|)^{-1 / 2-\varepsilon}$ and $R_{1}(z)$ $=\left(T+\Sigma V_{l}^{R}-z\right)^{-1}$, are bounded on $L^{2}(R)$. The latter can be deduced [35] from the results of R. Lavine [30], [31] (see also [15] and [32]).

On the other hand the results of [17] imply (see the remark at the beginning of the proof of Theorem VI.5) that an open neighborhood of the repulsive $L^{p} \cap L^{q}$. potentials produces strongly single channel systems.

Another class of potentials furnishing single channel systems is described by Simon [36] and Balslev [37] (see also [15]). These are dilation analytic potentials in the strip $|\arg \zeta|<\pi / 2$, continuous in $|\arg \zeta| \leqslant \pi / 2$.

Dilation analyticity. Let $U(\rho) f(x)=\rho^{-\nu(N-1) / 2} f\left(\rho^{-1} x\right)$. Then $U(\rho) T U(\rho)^{-1}=\rho^{2} T$ and $V_{l}(\rho) \equiv U(\rho) V_{l} U(\rho)^{-1}$ is the multiplication operator with $V_{l}\left(\rho^{-1} x^{l}\right)$. A $T$ bounded operator $V_{l}$ is said to be dilation analytic in $\theta \subset \mathbf{C ,} \theta \cap \mathbf{R} \neq \varnothing$ iff $V_{l}(\rho)$, considered as an operator from $H_{2}\left(R^{l}\right)=D\left(T^{l}\right)$ to $L^{2}\left(R^{l}\right)$, has analytic continuation into $\Theta$. In this case the family $H(\rho) \equiv U(\rho) H U(\rho)^{-1}$ has an analytic continuation from $\mathbf{R}$ to $\theta$ with the common domain $D(T)$. Note also that if $V_{l}$ is dilation analytic in $\theta$ it is dilation analytic in the sector $A=\{z \in \mathbf{C},|\arg z|<\alpha\}$, where $\alpha=\sup \{|\arg z|, z \in \mathcal{O}\}$. 
Spectral properties of $H$. Applying the results of the previous subsections to each operator $H^{a}=T^{a}+\Sigma V_{l}^{a}$ and taking into account Remark V.5, we obtain that

In particular, we have:

$$
\sigma_{\text {ess }}\left(H^{a}\right)=\bigcup_{b \subset a}\left[\sigma_{p}\left(H^{b}\right)+\sigma\left(T_{b}^{a}\right)\right] .^{1}
$$

(1) For a single channel, $N$-body system, $\sigma_{\text {css }}\left(H^{a}\right)=\sigma\left(T^{a}\right)=\overline{\mathbf{R}}^{+}$.

(2) If $H^{a}(\zeta)$ is the dilation family of Schrödinger operators, defined as above, then, applying the equation above to $\zeta^{-2} H^{a}(\zeta)=T^{a}+\Sigma_{l \subseteq a} \zeta^{-2} V_{l}^{a}(\zeta)$,

$$
\sigma_{\text {ess }}\left(H^{a}(\zeta)\right)=\bigcup_{b \subset a}\left[\sigma_{d}\left(H^{b}(\zeta)\right)+\zeta^{2} \overline{\mathbf{R}}^{+}\right]
$$

(the first Balslev-Combes theorem).

We mention also another Balslev-Combes theorem which we use below:

The isolated eigenvalues of $H^{a}(\zeta)$ are locally independent of $\zeta$ or, more precisely, they are independent of $\zeta$ as long as they stay away from $\sigma_{\text {ess }}\left(H^{a}(\zeta)\right)$.

A consequence of this fact is

$$
\begin{gathered}
\sigma_{d}\left(H^{a}(\zeta)\right) \subset \lambda_{\min }+2 \bar{A}^{ \pm} \text {for } \zeta \in A^{ \pm}, \text {where } \lambda_{\min }=\min \sigma_{d}\left(H^{a}\right) \\
\text { if } \sigma_{d}\left(H^{a}\right) \neq \varnothing \text { and } \lambda_{\min }=0 \text { otherwise and } A^{ \pm}=A \cap \mathbf{C}^{ \pm} .
\end{gathered}
$$

In passing, we remark that the first Balslev-Combes theorem implies that $\sigma_{\text {s.c. }}\left(H^{a}\right)=\varnothing$ for each $a \in \mathbb{Q}$.

In conclusion we mention the following fact [21]: In the case of a single channel system with potentials $V_{l}(\zeta) \in L^{p} \cap L^{q}\left(R^{\nu}\right), p>\nu / 2>q$, each $H^{a}(\zeta)$ has only a finite number of eigenvalues in any compact region of a neighborhood of $\mathbf{R}$.

\section{Scattering theory.}

THEOREM VI.5. Let $\mathrm{H}$ be the Hamiltonian of an $\mathrm{N}$-body, strongly single channel system with real, dilation analytic potentials $V_{l}$ such that $V_{l}(\zeta) \in L^{p} \cap L^{q}\left(\mathbf{R}^{\nu}\right)$, $p>\nu / 2>q$, for each $\zeta \in A$. Then for all $l$ and $s$ and $\zeta \in A$, $\left|V_{l}(\zeta)\right|^{1 / 2} R\left(z \zeta^{2}, \zeta\right)\left|V_{s}(\zeta)\right|^{1 / 2}$ is an analytic in $z \notin \cup_{a \neq a_{\max }}\left[\sigma_{d}\left(\zeta^{-2} H^{a}(\zeta)\right)+\overline{\mathbf{R}}^{+}\right]$ family of bounded operators on $L^{2}(R)$ with strong boundary values on $\overline{\mathbf{R}}^{+}$if $\operatorname{Im} z \cdot \operatorname{Im} \zeta>0$ and on

$$
\overline{\mathbf{R}}^{+} \cap\left[\mathbf{C} \backslash\left(\zeta^{-2} \bigcup_{a \neq a_{\max }} \sigma_{d}\left(H^{a}(\zeta)\right)+\overline{\mathbf{R}}^{+}\right)\right]
$$

if $\operatorname{Im} z \cdot \operatorname{Im} \zeta<0$. Moreover, $z$ is allowed to approach $\overline{\mathbf{R}}^{+}$with angles other than $\pi / 2$, e.g. $z=\lambda+i \varepsilon \zeta^{-2}, \varepsilon \rightarrow \pm 0$. In both cases $\left(z=\lambda+i \varepsilon\right.$ and $\left.z=\lambda+i \varepsilon \zeta^{-2}\right)$ the convergence is uniform in $\zeta$ from any compact subset of

for $\operatorname{Im} z \gtrless 0$.

$$
(\operatorname{Re} z)^{-1}\left[2 A \backslash[1, \infty) \bigcup_{a \neq a_{\max }} \sigma_{d}\left(H^{a}\left(e^{\mp i \alpha}\right)\right)\right]
$$

Proof. We prove only the first statement. The second statement requires a simple modification in Proposition VI.3. We will prove in fact a slightly weaker statement which covers only those strongly single channel systems for which there

\footnotetext{
${ }^{1}$ Remember that we use the convention that $\sigma_{d}\left(H^{a}\right)=\{0\}$ for $a=\{(1) \ldots(N)\}$.
} 
is a path in the complex $\left\{g_{l}\right\}$-space joining $\left\{g_{l}=1\right\}$ with $\left\{g_{l}=0\right\}$ and such that each of its points corresponds to a strongly single channel system (given a many-body system $\left\{T_{b}^{a}, V^{l}\right\}$ we associate with each point $\left\{g_{l}\right\}$ the many-body system $\left.\left\{T_{b}^{a}, g_{l} V^{l}\right\}\right)$. To prove the general case one can use the method of [17] which, instead of the analytic continuation in $g$, uses approximation of $\left(T^{a}-z\right) R^{a}(z)$ by the operators on $L^{2}\left(R^{a}\right)$ with smooth fast vanishing integral kernels. We use the method below because of a certain elegance it possesses and because a significant part of it can be formulated in an abstract way. In addition, we conjecture that for any given $L^{p} \cap L^{q}$-potential the set $\left\{\left(g_{l}\right): \sum g_{l} V_{l}\right.$ is strongly single channel $\}$ is simply complex connected and therefore contains a neighborhood of $\left(g_{l}=0\right)$.

It follows from Propositions VI.3 and VI.4 that conditions (i) and (ii) of Theorem V.7 are obeyed for the operator $\zeta^{-2} H(\zeta)=T+\zeta^{-2} V(\zeta)$. To verify condition (iii) we use Proposition IV.9. Since (iii $\left.{ }_{1}\right)$ is satisfied for dilation analytic potentials, and since $\sigma(T) \cap \sigma(T(\zeta))=\{0\}$ for $\operatorname{Im} \zeta \neq 0$, we have to check only (iii $)$ for one point $\lambda=0$. This is done in the following two lemmas. For simplicity we consider only the case $\nu \geqslant 3$.

Lemma VI.6a. Let $V^{l} \in L^{p} \cap L^{q}\left(\mathbf{R}^{\nu}\right), p>\nu / 2>q$. Then for all internal points of $G^{l}=\left\{g: \sigma_{p}\left(H^{l}(g)\right)=\varnothing\right\}$ the equation $H^{l}(g) \psi=0$ has no nontrivial solutions in $\left(T^{l}\right)^{-1}\left|V^{l}\right|^{1 / 2} L^{2}\left(R^{l}\right)$.

Proof. Let, on the contrary, $H^{l}(g) \psi=0$ with $\psi \in\left(T^{l}\right)^{-1} V^{l} L^{2}\left(R^{l}\right)$ and $\psi \neq 0$. Then $-g^{-1} \in \sigma\left(V^{l}\left(T^{l}\right)^{-1}\right)$ on $\left|V^{l}\right|^{1 / 2} L^{2}\left(R^{l}\right)$. By the perturbation theory (we use here the fact that $V^{l}\left(T^{l}-\lambda\right)^{-1}$ is norm continuous as $\left.\lambda \uparrow 0\right)$ for any sufficiently small $\lambda<0$ there exists $g^{\prime}$ such that $-g^{\prime-1} \in \sigma\left(V^{l}\left(T^{l}-\lambda\right)^{-1}\right)$ and $g^{\prime} \rightarrow g$ as $\lambda \rightarrow 0$. The latter implies that $\lambda \in \sigma_{d}\left(H^{\prime}\left(g^{\prime}\right)\right)$ for $g^{\prime}$ as close to $g$ as we wish. However, this is impossible since $g$ is an internal point of $G^{l}$.

Lemma VI.6b. Let the conditions of Theorem VI.5 be satisfied. Then $H^{a} \psi=0$, $\psi \in\left(T^{a}\right)^{-1} \Sigma\left|V_{l}\right|^{1 / 2} L^{2}\left(R^{a}\right)$, implies that either $\psi=0$ or $0 \in \sigma_{p}\left(H^{a}\right)$ for all a with $\nu(N-\#(a))>4$. Here \#(a) is the number of clusters in the partition a.

Proof. In the proof below we omit the superindex $a$. Let $\psi \in$ $T^{-1} \Sigma\left|V_{l}\right|^{1 / 2} L^{2}(R)$; then $F(0)^{-1} \psi \in \Sigma\left|V_{l}\right|^{1 / 2} L^{2}(R)$. If, moreover, $\psi$ is a solution to $H \psi=0$, then $\varphi=F(0)^{-1} \psi$ is a solution to $\phi+L(0) \phi=0$. On the other hand, we can show, as in [16], [19], that $L(0)$ is defined and compact and $T F(0)$ is bounded on $L^{p}(R), \frac{3}{2}-\varepsilon \leqslant p \leqslant 2$. Therefore by an abstract result of [16], [19] all the eigenfunctions of $L(0)$ on $\Sigma\left|V_{l}\right|^{1 / 2} L^{2}(R)$ belong also to $L^{p}(R), \frac{3}{2}-\varepsilon<p<2$. Thus $\psi=F(0) \phi \in T^{-1} L^{P}(R)$. Hence by Sobolev's potential theorem, $\psi \in L^{2}(R)$. Let now $\alpha \in C_{0}^{\infty}(\mathbf{R})$ and $\alpha(\lambda)=1$ for $|\lambda| \leqslant 1$. Then $\alpha(T) \psi \in D(T)$. Furthermore, since $(1-\alpha(T)) T^{-1}$ is bounded and maps $\mathcal{H}$ into $D(T),(1-\alpha(T)) T^{-1} T \psi \in$ $D(T)$.

Now all three conditions of Theorem V.8 are verified for the operator $\zeta^{-2} H(\zeta)$. In order to derive the statement of Theorem VI.5 from that of Theorem V.8 we make the following remarks: 
(a) The statement of Theorem V.8 is applicable strictly speaking only to the case when $\zeta^{-2} \sigma_{d}\left(H^{a}(\zeta)\right) \cap \mathbf{R}=\varnothing$ for all $a \neq a_{\max }$, i.e. to single channel systems. However, the proof of the theorem goes through without a change if the last condition fails, but we restrict the boundary values to the following part of the positive semiaxis:

$$
\overline{\mathbf{R}}^{+} \cap\left[\mathbf{C} \backslash\left(\zeta^{-2} \bigcup_{a \neq a_{\max }} \sigma_{d}\left(H^{a}(\zeta)\right)+\overline{\mathbf{R}}^{+}\right)\right],
$$

where the union extends only to the $a$ 's with $\sigma_{d}\left(H^{a}(\zeta)\right) \neq \varnothing$.

(b) If $\psi \in R_{0}(\lambda \pm i 0) \Sigma_{l \subseteq a}\left|V_{l}(\zeta)\right|^{1 / 2} L^{2}\left(R^{a}\right)$ obeys $H^{a}(\zeta) \psi=\zeta^{2} \lambda \psi$ for $\zeta \in A^{ \pm}$, then $\psi=0$. If the same is true for $\zeta \in A^{ \pm}$, then, if $\psi \neq 0, \zeta^{2} \lambda \in \sigma_{d}\left(H\left(e^{\mp i \alpha}\right)\right)$.

Indeed, the fact that $\psi \in R_{0}(\lambda \pm i 0) \Sigma_{l \subseteq a}\left|V_{l}(\zeta)\right|^{1 / 2} L^{2}\left(R^{a}\right)$ obeys $H^{a}(\zeta) \psi=$ $\zeta^{2} \lambda \psi$ implies by virtue of (II.3) that $\varphi=F^{a}\left((\lambda \pm i 0) \zeta^{2}, \zeta\right)^{-1} \psi \in \Sigma\left|V_{l}(\zeta)\right|^{1 / 2} L^{2}\left(R^{a}\right)$ satisfies $\varphi+L^{a}\left((\lambda \pm i 0) \zeta^{2}, \zeta\right) \varphi=0$. The latter, by virtue of Lemma AI.2, implies that, if $\varphi \neq 0$, then $-1 \in \sigma\left(L^{a}\left(\lambda \zeta, \zeta e^{\mp i \gamma}\right)\right), \gamma>0$. Therefore, going backward and using that $\lambda \zeta^{2} \in \bigcap_{b \subset a} \rho\left(H_{b}\left(\zeta e^{\mp i \gamma}\right)\right), \lambda \zeta^{2} \in \sigma_{d}\left(H\left(\zeta e^{\mp i \gamma}\right)\right)$. The latter is impossible for $\zeta \in A^{ \pm}$, since taking $\gamma=|\arg \zeta|$ we would get $\lambda \zeta^{2} \in \sigma_{d}(H)\left(\lambda \zeta^{2} \notin \mathbf{R}\right.$ and $H$ is selfadjoint). For $\zeta \in A^{\mp}$ we find, taking $\gamma=\alpha-|\arg \zeta|$, that $\lambda \zeta^{2} \in \sigma_{d}\left(H^{a}\left(e^{\mp i \alpha}\right)\right)$. This completes the proof of Theorem VI.5.

Before proceeding to the theorem on a structure of the $S$-matrix, we describe a fiber direct integral with respect to $T$. We define $\int^{\oplus} \mathcal{H}_{\lambda} d \lambda$ as $L^{2}\left(\overline{\mathbf{R}}^{+}, L^{2}(\Omega)\right.$ ), i.e. $\mathcal{F}_{\lambda}=L^{2}(\Omega)$. Here $\Omega$ is the unit sphere in $R$ (w.r. to the inner product defined at the beginning of the section). The unitary operator $\Pi=\int^{\oplus} \Pi_{\lambda} d \lambda$ from $\mathcal{H}$ to $\int^{\oplus} \mathcal{F}_{\lambda} d \lambda$ is defined as

$$
\begin{gathered}
\left(\Pi_{\lambda} f\right)(\omega)=C_{N} \lambda^{\gamma} \int e^{-i \sqrt{\lambda} \omega \cdot x} f(x) d x, \\
\gamma=(\nu(N-1)-2) / 4, \quad C_{N}=\frac{1}{2}(2 \pi)^{-\nu(N-1) / 2} .
\end{gathered}
$$

Obviously, $\Pi_{\lambda} T=\lambda \Pi_{\lambda}$ on $\mathscr{D}(T)$ and $\Pi_{\lambda}=\lambda^{-1 / 2} \Pi_{1} U(\sqrt{\lambda})$, where $U(\rho)$ is the dilation group defined above in this section.

THEOREM VI.7. Let the conditions of Theorem VI.5 be satisfied. Then:

(1) $W^{ \pm}$and $W^{ \pm *}$ exist as strong Abel limits, $W^{ \pm}$are isometric;

(2) $W^{ \pm}$are complete, i.e. $W^{ \pm} W^{ \pm *}=1-E_{p}$;

(3) The scattering operator and scattering matrix are unitary;

(4) $T_{\lambda}=s-\lim _{\epsilon \downarrow 0} \Pi_{\lambda} T(\lambda+i \varepsilon) \Pi_{\lambda}^{*}$, where, remember, $T(z)=V-V R(z) V$, exists as a strongly continuous $B\left(L^{2}(\Omega)\right)$-valued function of $\lambda \in \mathbf{R}$ and $S(\lambda)=\mathbf{1}_{\lambda}+2 \pi i T_{\lambda}$.

Proof. Theorems IV.1 and VI.5 imply all the statements (see also Remark IV.2 on the existence of $W^{ \pm}$), except for the strong continuity of $T_{\lambda}$.

LEMMA VI.8. Let $l$ be a pair of indices and $M$ the multiplication operator by $f\left(x^{l}\right)$, $f \in L^{p} \cap L^{q}\left(\mathbf{R}^{\nu}\right), p>\nu>q$, or $f \in L^{2}\left(\mathbf{R}^{\nu}\right)$. Then $\Pi_{s} M$ is a uniformly bounded family of operators from $L^{2}(\Omega)$ to $L^{2}(R)$, strongly continuous in $s$. A similar statement is true also for $M^{*} \Pi_{s}$. 
Proof. Let $f \in L^{p} \cap L^{q}, p>\nu>q$. Then the statement follows from Lemmas III.5 and III.8, Proposition VI.2, equation (VI.3) and strong continuity at $\rho=1$ of $U(\rho)$ on $L^{p}$ (different underlying spaces!). If $f \in L^{2}$, then the statement is obtained by the application of the Cauchy-Schwarz inequality to $M \Pi_{s}^{*} \varphi, \varphi \in L^{2}(\Omega)$, or $\Pi_{s} M u, u \in L^{2}(R)$, written explicitly as an integral.

This lemma implies readily the strong continuity of $T_{\lambda}$.

Throrem VI.9. Let all the conditions of Theorem VI.5 be satisfied. Then the scattering matrix $S(\lambda)$ has a meromorphic continuation into the sector

$$
\lambda \in 2 A \backslash[1, \infty] \bigcup_{a \neq a_{\max }} \sigma_{p}\left(H^{a}\left(e^{-i \alpha}\right)\right) .
$$

This continuation has poles in $A \cap \mathrm{C}^{-}$only at eigenvalues of $H\left(e^{-i \alpha}\right)$, i.e. at the points where the meromorphic continuation of $(u, R(z) v)$ (on dilation analytic vectors $u, v)$ from $\mathrm{C}^{+}$across $\sigma(H)$ into the second Riemann sheet has its poles. If $\alpha>\pi / 2$, then the poles of $S(\lambda)$ on the negative semiaxis occur at most at the eigenvalues of $H$.

Proof. We consider $T_{\lambda} \equiv \Pi_{\lambda} V \Pi_{\lambda}^{*}-\Pi_{\lambda} V R(\lambda+i 0) V \Pi_{\lambda}^{*}$. Let $A^{ \pm}=\mathbf{C}^{ \pm} \cap A$. Using (VI.3), we find: $\Pi_{\lambda} V \Pi_{\lambda}^{*}=\lambda^{-1} \Pi_{1} V(\sqrt{\lambda}) \Pi_{1}^{*}$. Therefore $\Pi_{\lambda} V \Pi_{\lambda}^{*}$ is analytic in $\lambda \in 2 A$, where, remember, $A$ is the sector of dilation analyticity of $V_{l}$. Next we obtain

$$
\Pi_{\lambda} V R(\lambda+i \varepsilon) V \Pi_{\lambda}^{*}=\lambda^{-1} \Pi_{1} V(\sqrt{\lambda}) R(\lambda+i \varepsilon, \sqrt{\lambda}) V(\sqrt{\lambda}) \Pi_{1}^{*} .
$$

The r.h.s. is a $B\left(L^{2}(\Omega)\right)$-valued function, meromorphic in $\lambda \in 2 A$ as long as

$$
\lambda+i \varepsilon \notin \sigma_{\text {ess }}(H(\sqrt{\lambda}))=\bigcup_{a \neq a_{\max }}\left[\sigma_{d}\left(H^{a}(\sqrt{\lambda})\right)+\lambda \overline{\mathbf{R}}^{+}\right] .
$$

It follows from Theorem VI.5 and Lemma VI.8 that (VI.4) converge in the $L^{2}(\Omega)$-operator norm as $\varepsilon \downarrow 0$, uniformly in $\lambda$ from any compact subset of

$$
B \equiv 2 A \backslash[1, \infty] \bigcup_{a \neq a_{\max }} \sigma_{d}\left(H^{a}\left(e^{-i \alpha}\right)\right)
$$

(take $z=1+i \varepsilon \lambda^{-1}$ and $\zeta=\sqrt{\lambda}$ in Theorem VI.5). Then by the theorem on uniform convergence of analytic functions, the boundary value of (VI.4) as $\varepsilon \downarrow 0$ is a meromorphic function in $B$ which can have poles only where $R(\lambda+i 0, \sqrt{\lambda})$ does. All the poles of the latter family are in $A^{-}$and at the eigenvalues of $H\left(e^{-i \alpha}\right)$. The converse is also true. Indeed, for $\phi, F$, two dilation vectors, and $\lambda \in 2 A$ we have $\langle\phi, R(\lambda+i 0, \sqrt{\lambda}) F\rangle=\langle\phi(\bar{\zeta}), R(\lambda, \sqrt{\lambda} \zeta) F(\zeta)\rangle$, where $\zeta \in A^{-}$and $\phi(\zeta)$ is an analytic continuation of $U(\rho) \phi$. If $\lambda \in 2 A^{+}$, then taking $\zeta=\lambda^{-1 / 2} \in A^{-}$we see that the r.h.s. of this equality has no poles at all. If $\lambda \in 2 A^{-}$, we can take $\zeta=\lambda^{-1 / 2} e^{-i \alpha} \in A^{-}$to convince ourselves that it has poles exactly at eigenvalues of $H\left(e^{-i \alpha}\right)$.

VII. Operators $W(T-z)^{-1} U$. In this section we prove Proposition VI.3. The superindex $a$ is omitted in what follows. Our task is to show that the family $W(T-z)^{-1} U$ is bounded on $L^{2}(R)$ for $z \in \mathbf{C} \backslash \overline{\mathbf{R}}^{+}$, strongly continuous as $z$ approaches $\overline{\mathbf{R}}^{+}$and obeys the corresponding estimate. 
We study $W R_{0}(z) U$ using the following representation for $R_{0}(z)$ :

$$
R_{0}(z)=-i \int_{0}^{\infty} e^{i z t} e^{-i T t} d t, \quad z \in \mathbf{C}^{ \pm},
$$

valid for any selfadjoint $T$. We consider below two cases:

(i) $l \cap s=\varnothing$. Using the equation $T=T^{s}+T_{s}$ and the fact that $T_{s}$ commutes with $W$ we find $\left(\alpha^{-1}+\alpha^{-1}=1\right)$

$$
\left\|W e^{-i T t} U\right\| \leqslant\|U\|_{L^{2}\left(R^{s}\right) \rightarrow L^{\alpha^{\prime}}\left(R^{s}\right)}\left\|e^{-i T t}\right\|_{L^{\alpha^{\prime}}\left(R^{s}\right) \rightarrow L^{\alpha}\left(R^{s}\right)}\|W\|_{L^{\alpha}\left(R^{s}\right) \rightarrow L^{2}\left(R^{s}\right)} .
$$

Writing the kernel of $\exp \left(-i T^{s} t\right)$ as

$$
\text { const } \cdot t^{-\nu / 2} e^{-i x^{2} / t} e^{-i x y / t} e^{-i y^{2} / t}
$$

and using the boundedness of the Fourier transform from $L^{p}$ to $L^{p^{\prime}}, p<2$, we obtain the estimate $\left\|e^{-i T^{s} t}\right\|_{\alpha \rightarrow \alpha^{\prime}} \leqslant$ const $\cdot t^{-\nu(1 / 2-1 / \alpha)}$. Taking into account this estimate and

$$
\|W\|_{L^{\alpha}\left(R^{s}\right) \rightarrow L^{2}\left(R^{s}\right)} \leqslant\|\psi\|_{r} \text { and }\|U\|_{L^{2}\left(R^{s}\right) \rightarrow L^{\alpha^{\prime}\left(R^{s}\right)}} \leqslant\|\varphi\|_{r}
$$

$\alpha^{\prime-1}=2^{-1}+r^{-1}$ (which follows from $\|f \cdot u\|_{\alpha} \leqslant\|f\|_{r}\|u\|_{\beta}, \alpha^{-1}=r^{-1}+\beta^{-1}$ ), we finally arrive at

$$
\left\|W e^{-i t T} U\right\| \leqslant \text { const }\|\psi\|_{r}\|\varphi\|_{r} t^{-3 / r} .
$$

This implies the desired properties of $W R_{0}(z) U$.

(ii) $l \cap s=\varnothing$. In this case we use the decomposition $T=T^{l}+T^{s}+T_{b}$, $b=l \cup s$. Since $T^{s} / T^{l}, T_{b}$ commutes with $U / W$, we have

$$
W e^{-i T t} U=e^{-i T_{b} t} e^{-i T^{t}} U W e^{-i T^{t} t} \text {. }
$$

We prove now the strong continuity of $W R_{0}(z) U$. The estimate of its norm follows in the same way. To spare us unpleasant domain remarks, we use henceforth the extended definition of the norm: $\|A u\|=\infty$ if $u \notin \mathscr{D}(A)$ and $\|A\|=\infty$ if $A$ is unbounded. Using (VII.1) and (VII.2) we obtain

$$
\begin{aligned}
\| W\left(R_{0}\left(z^{\prime}\right)-\right. & \left.R_{0}(z)\right) U f \| \leqslant \sup _{\|\varphi\|=1} \int\left|\left\langle W e^{-i T t} U f, \varphi\right\rangle\right|\left|e^{i z z^{\prime} t}-e^{i z t}\right| d t \\
& \leqslant\left\{\int\left\|W e^{-i T^{s} t} f\right\|^{2}\left|e^{i z^{\prime} t}-e^{i z t}\right|^{2} d t\right\}^{1 / 2} \sup _{\|\varphi\|=1}\left\{\int\left\|U^{*} e^{i T T^{t}} \varphi\right\|^{2} d t\right\}^{1 / 2} .
\end{aligned}
$$

To complete our estimate we use Kato's inequality [11]:

$$
\int \frac{\left\|A e^{i T t} u\right\|^{2}}{\|u\|^{2}} d t \leqslant \sup _{\varepsilon>0}\left\|A \delta_{\varepsilon}(T-\lambda) A^{*}\right\|,
$$

valid for any selfadjoint $T$ and densely defined closed $A$, and the estimates of case (i) for $U^{*} R_{0}(z) U$ and $W R(z) W^{*}$.

VIII. Boundary values of $\Pi\left[W_{l_{i}}(T-z)^{-1} U_{l_{i+1}}\right]$. In this section we prove Proposition VI.4. It states that the product of three $a$-connected graphs on $L^{2}\left(R^{a}\right)$ is norm continuous as $\operatorname{Im} z \rightarrow \pm 0$. In virtue of Theorem I.2 this implies that the corresponding boundary values are compact. By the graph on $L^{2}\left(R^{a}\right)$ we understand here an operator of the form $\Pi\left[W_{l_{i}}\left(T^{a}-z\right)^{-1} U_{l_{i+1}}\right]$, where, remember, $U_{l}$ and $W_{l}$ denote the multiplication operators by functions $\varphi_{l}\left(x^{l}\right)$ and $\psi_{l}\left(x^{l}\right)$, respectively, $\varphi_{l}$, 
$\psi_{l} \in\left(L^{p} \cap L^{q}\right)\left(\mathbf{R}^{\nu}\right), p>\nu>q$. We say that the graph is $a$-connected if $\cup l_{i}=a$. The proof is based on the two following lemmas.

LEMMA VIII.1. Graphs $\Pi\left[W_{l_{l}}\left(T^{a}-z\right)^{-1} U_{l_{i+1}}\right]$ are norm continuous in $\varphi_{l}, \psi_{l} \in L^{p}$ $\cap L^{q}\left(\mathbf{R}^{\nu}\right), p>\nu>q$, uniformly in $z \in \mathbf{C} \backslash \overline{\mathbf{R}}^{+}$.

Proof. The statement follows from the estimate of Proposition VI.3.

LEMMA VIII.2. The product of three a-connected graphs with $\varphi_{l}, \psi_{l} \in S\left(\mathbf{R}^{v}\right)$ is norm continuous on $L^{2}\left(R^{a}\right)$ as $\operatorname{Im} z \rightarrow \pm 0$.

Before proceeding to the proof of this lemma we deduce the proof of Proposition VI.4 from Lemmas VIII.1 and VIII.2. Indeed, since $S$ is dense in $L^{p}$, there exist sequences $\varphi_{l}^{(n)}$ and $\psi_{l}^{(n)}$ converging in $L^{p} \cap L^{q}\left(\mathbf{R}^{\nu}\right), p>\nu>q$, to $\varphi_{l}$ and $\psi_{l}$, respectively. Given a graph $G$ we construct the new graphs, $G^{(n)}(z)$, by replacing in $G(z)$ all $U_{l}$ and $W_{l}$ by the operators $U_{l}^{(n)}$ and $W_{l}^{(n)}$ of multiplication by $\varphi_{l}^{(n)}\left(x^{l}\right)$ and $\psi_{l}^{(n)}\left(x^{l}\right)$, respectively. By Lemma VIII.1, $G^{(n)}(z) \rightarrow G(z)$ is norm, uniformly in $z \in \mathbf{C} \backslash \overline{\mathbf{R}}^{+}$, as $n \rightarrow \infty$. Now consider the product of three $a$-connected graphs and the norm approximation to this product constructed as above. By Lemma VIII.2, this approximation is norm continuous as $\operatorname{Im} z \rightarrow \pm 0$. Hence the product itself is norm continuous as $\operatorname{Im} z \rightarrow \pm 0$. This completes the proof of Proposition VI.4.

Proof of Lemma VIII.2. Below we prove a statement stronger than Lemma VIII.2. Namely, we show that the Fourier transform of the kernel of the product in Lemma VIII. 2 is a Hölder continuous, fast decreasing at infinity function, Hölder continuous in the parameter $z \in \mathbf{C} \backslash \overline{\mathbf{R}}^{+}$up to $\overline{\mathbf{R}}^{+}$.

We set

$$
\Delta_{x}^{\nu}(h) f(x, y)=|h|^{-\nu}(f(x+h, y)-f(x, y)) \text { if } 0<\nu \leqslant 1
$$

and

$$
\Delta_{x}^{v}(h) f(x, y)=f(x, y) \text { if } \nu=0 .
$$

LEMMA VIII.3. Let $G(z)$ be a product of three a-connected graphs with $\varphi_{l}$, $\psi_{l} \in S\left(\mathbf{R}^{\nu}\right)$. Then the Fourier transform, $G(p, q, z)$, of its kernel satisfies the estimate (VIII.1) $\left|\Delta_{p, q}^{\nu}(h) \Delta_{z}^{\mu}(w) G(p, q, z)\right|<$ const $\cdot(1+|p-q|)^{-r}, \quad r \in \mathbf{R}^{+}$.

Here $p$ and $q$ are two sets of independent variables in the space dual to $R^{a}$ (i.e. in the corresponding momentum space).

Proof. The expression for the kernel of $G(z)$ in the momentum representation (i.e. the Fourier transform of the kernel) can be easily computed, since the kernels of $U_{l}, W_{l}$ and $\left(T^{a}-z\right)^{-1}$ in this representation are known. It has the form

$$
G(p, q, z)=\int \frac{\phi(p, q, k) d^{m} k}{\prod_{1}^{s}\left[P_{i}(p, q, k)-z\right]},
$$

where $\phi(p, q, k) \in C^{\infty}$ comes from the potential part $\left(U_{l}\right.$ and $\left.W_{l}\right)$ of $G(z)$ and $P_{i}(p, q, k)$ is the symbol of $T^{a}$ expressed in the variables $p, q, k$, using an 
$i$-dependent linear function. The estimate of the decay of $G(p, q, z)$ at infinity can be easily obtained if we note that those of the $P_{i}$ 's with large enough $p_{k}$ or $q_{k}$ (say $\left.p_{k}^{2}>10 \operatorname{Re} z+1\right)$ are not singular in the sense that $P_{i}-\operatorname{Re} z>\delta>0$. An estimation of the decay of $G(p, q, z)$ in such a $p_{k}$ or $q_{k}$ is a rather simple but, unfortunately, boring and long exercise. Since, moreover, the precise form of the estimating function is not important (what is important is its $L^{1}$-property) we omit here the derivation of the infinity-decay estimate.

To obtain the smoothness estimates for those variables $p_{j}$ and $q_{j}$ which stay in the bounded region of $\mathbf{R}^{\nu}$ and the smoothness estimates in $z$, we join those $P_{i}$, which contain variables from the vicinity of infinity specified above, to $\phi$.

The resulting integral is of the form

$$
J(u, z)=\int \frac{\phi(k) d^{m} k}{\Pi_{1}^{s}\left[\left(x, R^{i} x\right)-z\right]}, \quad x=(k, u), \quad k \in \mathbf{R}^{\nu m}, \quad u \in \mathbf{R}^{\nu n},
$$

where $u$ varies in a compact region of $\mathbf{R}^{\nu n}, \phi \in C^{\infty}\left(\mathbf{R}^{\nu m}\right)$ and satisfies $\left|(1+|k|)^{r} D^{\alpha} \phi(k)\right| \leqslant$ const for all $\alpha$ and some $r>0$ and $R^{i}$ are real, nonnegative, $(m+n) \times(m+n)$-matrices. $R^{i}$ act on the space $\mathbf{R}^{\nu(m+n)}$, of which vectors are written as $p=\left(p_{i} \ldots p_{m+n}\right), p_{i} \in \mathbf{R}^{\nu}$, according to the equation $(R p)_{i}=$ $\sum_{j=1}^{m+n}(R)_{i j} p_{j}$.

It is shown in Lemma III.1 of [17] that such integrals are Hölder continuous in $u$ and $z$ (including $z \rightarrow \mathbf{R}$ ). The conditions of this lemma are satisfied by (VIII.2). For the reader's convenience we reproduce the lemma under more restrictive conditions, which are still obeyed in our case, in a separate preprint which can be obtained from the author.

Here we mention only that to obtain the desired estimates on (VIII.3) we first use the Feynman identity,

$$
\prod_{i=1}^{s} A_{i}^{-1}=\int_{[0,1]^{s}}\left(\sum_{s} \alpha_{i} A_{i}\right)^{-s} \delta\left(1-\sum \alpha_{i}\right) d^{s} \alpha,
$$

to transform the product of $s$ polynomials (of the second degree) in the denominator into one polynomial (also of the second degree) but taken to the sth power. Then we integrate by parts in $k$.

IX. Analyticity of the wave operators. In this section we show that the wave operators $U(\rho) W^{ \pm} U(\rho)^{-1}$ have analytic continuations, $W^{ \pm}(\zeta)$, from $\overline{\mathbf{R}}^{+}$to the angle $A$ with the cuts along the semilines

$$
E=\overline{\mathbf{R}}^{+} \bigcup_{a \neq a_{\max }} \sigma_{d}\left(H\left(e^{ \pm i \alpha}\right)\right)^{1 / 2},
$$

where, remember, $\alpha=\sup \{|\arg z|, z \in A\}$. In order to construct $W^{ \pm}(\zeta)$ we need a scattering theory for nonselfadjoint operators which is outlined below.

First let $T$ be any absolutely continuous operator and $H$ any operator on the same Hilbert space $\mathcal{H}$. To define the wave operators we use stationary expressions (III.3). However, we have to require that the domains of $Z^{(e)}(\Delta)$ contain a common dense set, on which we define the limit. Note that in the nonselfadjoint case some of the properties of $W^{ \pm}$and $Z^{ \pm}$, discussed in §III, fail: $Z^{ \pm}(\Delta) \neq W^{ \pm}(\Delta)^{*}$ and 
$W^{ \pm}(\Delta)$ and $Z^{ \pm}(\Delta)$ are not partially isometric in general, and the other properties (e.g. intertwining) are not implied any more by the mere existence of $W^{ \pm}(\Delta)$ and $Z^{ \pm}(\Delta)$.

LEMMA IX.1. Let $W^{ \pm}(\Delta)$ and $Z^{ \pm}(\Delta)$ exist in the weak sense. Then $E_{p} W^{ \pm}(\Delta)=$ $Z^{ \pm}(\Delta) E_{p}=0$.

Proof. Consider some eigenvalue $\lambda \in \sigma_{p}(H)$. If $\lambda \notin \Delta$, then the statement is obvious. If $\lambda \in \Delta$, then the problem is reduced to the selfadjoint case.

LEMMA IX.2. $Z^{(e)}(\Delta)$ are defined on all vectors $u \in \mathcal{H}$ such that

$$
|\varepsilon| \int\|R(\lambda+i \varepsilon) u\|^{2} d \lambda<\infty
$$

and

$$
\left\|Z^{(e)}(\Delta) u\right\| \leqslant\left\{\frac{\varepsilon}{\pi} \int_{\Delta}\|R(\lambda+i \varepsilon) u\|^{2} d \lambda\right\}^{1 / 2} .
$$

The same statement holds also for $W^{(e)}(\Delta)^{*}$.

Proof. Consider $Z^{(e)}(\Delta)$. Applying the Schwarz inequality to

$$
\varepsilon \int_{\Delta}\left|\left(R(\lambda+i \varepsilon) u, R_{0}(\lambda+i \varepsilon) v\right)\right| d \lambda
$$

and using the fact that $|\varepsilon| \int\left\|R_{0}(\lambda+i \varepsilon) v\right\|^{2} d \lambda=\pi\|v\|^{2}$, we find (IX.1).

COROLlary IX.3. Let $Z^{ \pm}(\Delta)$ exist. Consider the set of all vectors $u$ for which the supremum of the RHS of (IX.1) over $|\varepsilon|>0$ is finite. Then $Z^{ \pm}(\Delta)$ is defined on this set and obeys

$$
\left\|Z^{ \pm}(\Delta) u\right\| \leqslant \sup _{\varepsilon \in \mathbf{R}^{ \pm}}\left\{\frac{|\varepsilon|}{\pi} \int_{\Delta}\|R(\lambda+i \varepsilon) u\|^{2} d \lambda\right\}^{1 / 2} .
$$

The same is true for $W^{ \pm}(\Delta)^{*}$.

Lemma IX.4. Assume that $Z^{ \pm}(\Delta) / W^{ \pm}(\Delta)$ exist (in the weak sense) and

$$
\mathrm{w}-\lim \varepsilon \int_{\Delta} R(\lambda+i \varepsilon) u d \lambda=0 \text { on a dense set. }
$$

Then $Z^{ \pm}(\Delta) / W^{ \pm}(\Delta)$ are intertwining for $(H, T): T Z^{ \pm}(\Delta)=Z^{ \pm}(\Delta) H, H W^{ \pm}(\Delta)=$ $W^{ \pm}(\Delta) T$.

Proof. Consider $W^{ \pm}(\Delta)$. Applying twice $A R_{A}(z)=1+z R_{A}(z)$, we find

$$
H W^{(e)}(\Delta)-W^{(e)}(\Delta) T=\frac{\varepsilon}{\pi} \int\left[R_{0}(\lambda-i \varepsilon)-R(\lambda+i \varepsilon)\right] d \lambda+2 i \varepsilon W^{(e)}(\Delta) .
$$

Now, $\varepsilon \int R_{0}(\lambda-i \varepsilon) d \lambda$ vanishes as $|\varepsilon| \rightarrow 0$ since $T$ is selfadjoint and the other terms on the right-hand side vanish by the assumptions.

REMARK IX.5. (IX.3) is obviously obeyed for those $u$ 's for which the RHS of (IX.2) is finite. 
THEOREM IX.6. Theorems III.2 and IV.1 remain true if the condition of selfadjointness of $H$ is replaced by the weaker one: $\sigma_{\text {ess }}(H) \subset \mathbf{R}$ (i.e. $V_{a}$ are allowed to be nonselfadjoint in Theorem IV.1). $E(\Delta)$ in the statement of the theorems is defined as $E(\Delta)=\lim _{e \downarrow 0} \int_{\Delta} \delta_{e}(H-\lambda) d \lambda$ and $Z^{(e)}(\Delta)$ converge strongly to $Z^{ \pm}(\Delta)$ as bounded operators from $X \equiv \Sigma\left|V_{a}\right|^{1 / 2} \mathcal{H}$ to $\mathcal{H}$ (we have, in addition, to assume in Theorem III.2 that $Y=X$ and $(H-T) R(\lambda+i \varepsilon)$ extends to a family of bounded operators from $X$ to $\left.L^{2}(\Delta, X)\right)$.

REMARK IX.7. Applying Theorem IX.6 to $H^{*}$ we obtain the same statement for $W^{ \pm}(\Delta)^{*}$.

Proof. The selfadjointness of $H$ is not used in an essential way in the proofs of Theorems III.2 and IV.1. The same proofs go through if one requires instead that $\sigma_{\text {ess }}(H) \subset \mathbf{R}$.

We proceed now to the many-body systems. Let $U_{l}$ be the multiplication operators by functions $\varphi_{l}\left(x^{l}\right), \varphi_{l} \in L^{p} \cap L^{q}\left(\mathbf{R}^{\nu}\right), p>\nu>q$.

TheOREM IX.8. Let $H$ be the many-body Hamiltonian with real, dilation analytic potentials $V_{l}$ such that $V_{l}(\zeta) \in L^{p} \cap L^{q}\left(\mathbf{R}^{\nu}\right), p>\nu / 2>q$, and let $\sigma_{p}\left(H^{a}\right)=\varnothing$ for $a \neq a_{\max }$. Then (1) the wave operators $Z^{ \pm}(\zeta, \Delta)$ and $W^{ \pm}(\zeta, \Delta), \zeta \in A \backslash E$, for the pair $\left(\zeta^{-2} H(\zeta), T\right)=\left(H(\zeta), \zeta^{2} T\right)$ exist as strong limits of bounded operators from $\Sigma U_{l} L^{2}(R)$ to $L^{2}(R)$ and from $L^{2}(R)$ to $\Sigma U_{l} L^{2}(R)$, respectively; (2) $W^{ \pm}(\zeta, \Delta) Z^{ \pm}(\zeta, \Delta)=E\left(\zeta, \Delta \cap \Delta^{\prime}\right)$ and $Z^{ \pm}(\zeta, \Delta) W^{ \pm}\left(\zeta, \Delta^{\prime}\right)=E_{0}\left(\Delta \cap \Delta^{\prime}\right)$; (3) $Z^{ \pm}(\zeta, \Delta)$ and $W^{ \pm}(\zeta, \Delta)$ for $\Delta \cap \zeta^{-2} \sigma_{p}(H(\zeta))=\varnothing$ are analytic in $\zeta \in A \backslash E$; (4) $Z^{ \pm}(\rho, \Delta)=U(\rho) Z^{ \pm}(\Delta) U(\rho)^{-1}, \rho \in \mathbf{R}$, and the same relation holds also for $W^{ \pm}(\rho, \Delta)$.

COROLlary IX.9. The local scattering operator $S(\zeta, \Delta)=Z^{+}(\zeta, \Delta) W^{-}(\zeta, \Delta)$ for $\left(\zeta^{-2} H(\zeta), T\right)$ and $\Delta \cap \sigma_{p}\left(\zeta^{-2} H(\zeta)\right)=\varnothing$ is analytic in $A \backslash E$ and $S(\rho, \Delta)=$ $U(\rho) S \Delta U(\rho)^{-1}$ for $\rho \in \overline{\mathbf{R}}^{+}$.

Proof OF Theorem IX.8. (1) and (2) follow from Theorems VI.5 and IX.6. By the way, (2) follows also from (1) and (3). Equation (III.3) shows that $Z^{(e)}(\zeta, \Delta)$, considered as a family of operators from $\Sigma U_{l} L^{2}(R)$ to $L^{2}(R)$, is analytic in $\zeta$ as long as $\zeta$ stays away from $E$. The analyticity of $Z^{ \pm}(\zeta, \Delta)$ in $A \backslash E$ follows from the analyticity of $Z^{(e)}(\zeta, \Delta)$ and the fact that the convergence of $Z^{(e)}(\zeta, \Delta)$ to $Z^{ \pm}(\zeta, \Delta)$ is uniform in $\zeta$ from any compact subset of $A \backslash E$. The latter can be seen from a careful examining of the proof of (1).

Appendix I. Regularizers. In this appendix we present a simple application of the regularizers to the study of spectral properties of operators. In the sequel $H$ and $G$ denote an operator (in general unbounded and nonselfadjoint) on a Hilbert space $\mathcal{H}$ and an open set in $\mathbf{C}$.

THEOREM AI.1. Let there exist a family $F(z): G \rightarrow B(\mathcal{H}, \mathscr{D}(H))$ with a bounded inverse $F(z)^{-1}: G \rightarrow B(\mathscr{D}(H), \mathcal{H})$, such that the operator $(H-z) F(z)-1$, raised to some power, is compact on $\mathcal{H}$. Then $\sigma_{\text {ess }}(H) \subset \mathbf{C} \backslash G$. 
REMARK. This theorem can be regarded as a generalization of Weyl's- $\varepsilon$ theorem. Indeed, let $H=T+V$, where $V$ is $T$-compact. Taking $F(z)=(T-z)^{-1}$ for $z \in \rho(T)$ we see immediately that the conditions of Theorem AI.1 are satisfied and therefore $\rho(T) \cap \sigma_{\text {ess }}(H)=\varnothing$. Reversing the role of $H$ and $T$ we get $\rho(H) \cap$ $\sigma_{\text {ess }}(T)=\varnothing$. If $\sigma_{d}(T)=\varnothing$, then $\sigma_{\text {ess }}(H)=\sigma(T)$.

Proof. Introduce the operator $A(z)=(H-z) F(z)$. Then

$$
H-z=A(z) F(z)^{-1} \text {. }
$$

Therefore in virtue of the properties of $F(z), \operatorname{Ker}(H-z)=F(z) \operatorname{Ker} A(z)$.

Since $A(z)-1$, raised to some power, is compact, $A(z) \uparrow \mathcal{H} \ominus \operatorname{Ker} A(z)$ has a bounded inverse on $\mathcal{H}$. Therefore, in virtue of (AI.1), so has $H-z$, restricted to $\mathscr{D}(H) \ominus F(z) \operatorname{Ker} A(z)$. Since $\operatorname{dim} \operatorname{Ker} A(z)<\infty$, the latter conclusion implies that $z \notin \sigma_{\text {ess }}(H)$.

Lemma AI.2. Let $G \subset \mathbf{C} \backslash \sigma_{\text {ess }}(H)$ and let $F(z)$ obey the conditions of Theorem AI.1. Let there exist a Banach space $X$ such that $X \cap \mathcal{H}$ is dense in $X$ and $\mathcal{H}$ and:

(1) $(H-z) F(z)-1$ is bounded on $X$ and strongly continuous as $z \rightarrow \partial G$ and, raised to some power, is continuous and compact for $z \in \bar{G}$.

Assume the following condition is satisfied:

(2) There is a unitary representation, $\rho \rightarrow U(\rho)$, of $\overline{\mathbf{R}}^{+}$on $\mathcal{H}$ such that $U(\rho) H U(\rho)^{-1}$ and $U(\rho) A(z) U(\rho)^{-1}$ have analytic continuations, $H(\zeta)$ and $A(z, \zeta)$, to a domain $\theta \subset \mathbf{C}, \mathcal{O} \cap \overline{\mathbf{R}}^{+} \neq \varnothing$, and $A(z, \zeta)$ is continuous in $z \in \bar{G} \backslash \sigma_{\text {ess }}(H(\zeta))$ and in $\zeta \in \mathcal{O}$ for $z \in G$ on $\mathcal{H}$ and on $X$.

Then $0 \in \sigma(A(z)) \Leftrightarrow z \in \sigma_{p}(H)$ for $z \in \bar{G} \backslash \sigma_{\text {ess }}(H(\zeta)), \operatorname{Im} \zeta \neq 0$.

Proof. In order to avoid lengthy expressions we assume here that $A(z)-1$ is compact and norm continuous itself (and not only in a power). We begin with

Lemma AI.3. Let $Q, D \subset \mathbf{C}$ and $K(z, \zeta)$ a family of compact operators, norm continuous in $z \in \bar{Q}$ for all $\zeta \in \bar{D}$ and analytic in $\zeta \in \bar{D}$ as $z \in Q$. Let, moreover, $K(z, \zeta)=U(|\zeta|) K\left(z, e^{i \arg \zeta}\right) U(|\zeta|)$, where $U(\rho)$ is unitary for $\rho \in \mathbf{R}^{+}$. Then for $z \in \bar{Q}$ and $\nu \neq 0$,

$$
\nu \in \sigma(K(z, \rho)), \quad \rho \in \partial D, \quad \Leftrightarrow \nu \in \sigma(K(z, \zeta)), \quad \zeta \in D .
$$

Proof. For $z \in Q$ the statement is obtained by the standard Combes argument: $\sigma(K(z, \zeta))$ is analytic in $\zeta^{1 / p}$ for some integer $p>0$ (by the analytic perturbation theory) and is, at the same time, independent of $|\zeta|$ (by the condition of the theorem). Therefore it is $\zeta$-independent.

Consider now $z \in \partial G$. We use

Lemma AI.4. Let $K(\lambda)$ be a continuous family of compact operators, $\lambda \in Q, a$ closed set, and $\nu \in \sigma\left(K\left(\lambda_{0}\right)\right)$. Then there exist a neighborhood $V$ of $\lambda_{0}$ and $\nu(\lambda) \in$ $\sigma(K(\lambda))$ continuous in $\lambda \in V \cap Q$, for which $\nu\left(\lambda_{0}\right)=\nu$.

Proof. For any contour $\Gamma \subset \rho\left(K\left(\lambda_{0}\right)\right)$ around the point $\nu$ there is a small disk, $U$, with the center at $\lambda_{0}$, such that $\Gamma \subset \rho(K(\lambda))$ for $\lambda \in U$. 
Therefore we can write $P_{\lambda}=(2 \pi i)^{-1} \phi_{\Gamma}(z-K(\lambda))^{-1} d z$ for $\lambda \in U$. Since $P_{\lambda}$ is continuous in $\lambda \in U$ and $P_{\lambda_{0}} \neq 0$, we have $P_{\lambda} \neq 0$, for $\lambda \in U$. Therefore $\sigma(K(\lambda))$ $\cap V \neq \varnothing$ for $\lambda \in U$ and the neighborhood $V$ with $\partial V=\Gamma$.

We proceed to the proof of Lemma AI.3. Assume for $z_{0} \in \partial Q, \nu \in \sigma(K(z, \rho))$. By Lemma AI.4, there is $\nu(z)$, continuous in a $Q$-neighborhood of $z_{0}$ and such that $\nu\left(z_{0}\right)=\nu$ and $\nu(z) \in \sigma(K(z, \rho))$. Since $\sigma(K(z, \zeta))$ is independent of $\zeta$ as long as $K(z, \zeta)$ is analytic in $\zeta$ (see above), $\nu(z) \in \sigma(K(z, \zeta))$. Therefore, in virtue of the norm continuity of $K(z, \zeta)$ in $z \in Q$ for $\zeta \in D, \nu \in \sigma\left(K\left(z_{0}, \zeta\right)\right.$ ) (see Appendix to [16).

To prove the opposite direction we repeat our arguments in the reverse order.

Further, the proof of Lemma AI.2 goes as follows. Applying Lemma AI.3 to $A(z)-\mathbf{1}$, we find that $0 \in \sigma(A(z)) \Leftrightarrow 0 \in \sigma(A(z, \zeta))$ for $z \in \partial G \backslash \sigma_{\text {ess }}(H(\zeta))$, $\operatorname{Im} \zeta$ $\neq 0$. Since $A(z, \zeta)-\mathbf{1}$ is norm continuous in $z \in \bar{G}$ as long as $z \notin \sigma_{\text {ess }}(H(\zeta))$ and is compact on $\mathcal{H}$ and on $X$ for $z \in G$, it is also compact for $z \in \partial G \backslash \sigma_{\text {ess }}(H(\zeta))$ ). Next, we use the lemma from [17], [19], stating that if a compact operator $K$ is defined on Banach spaces $X$ and $Y$ and $Y \subset X$, densely and continuously, then the spectra of $K$ on $X$ and on $Y$ coincide. We apply this lemma to $X \cap \mathcal{H}, X, \mathcal{H}$ and $A(z, \zeta)-1$ to conclude that $\sigma(A(z, \zeta))$ on $X$ and on $\mathcal{H}$ is the same. This and the definition of $A(z, \zeta)$ produce: $0 \in \sigma(A(z, \zeta)) \Leftrightarrow z \in \sigma_{d}(H(\zeta))$. Finally, $z \in \sigma_{d}(H(\zeta)) \Leftrightarrow z \in$ $\sigma_{d}(H)$ for $z \in \partial G \cap\left(\mathbf{C} \sigma_{\text {css }}(H(\zeta))\right)$ by Lemma IV.11.

THEOREM AI.5. Assume in addition to the conditions of Lemma AI.12 that for some family of operators $T(z)$ with $\mathscr{D}(T(z)) \subset \mathcal{D}(H), T(z) F(z)$ extends to a family of bounded operators on $X$, strongly continuous in $z \in \bar{G}$. Then $T(z)(H-z)^{-1}$ extends to a family of bounded operators on $X$, strongly continuous in $z \in \bar{G} \backslash\left(\bar{G} \cap \sigma_{p}(H)\right)$.

Proof. The proof follows from Lemma AI.2.

Appendix II. Proof of Theorem III.2. Before proceeding to the proof of the theorem we mention a few auxiliary relations which follow from the density of $X \cap \mathcal{H}$ in $X$ and some facts about operator-valued functions [19]. Let $T$ be an absolute continuous operator and $x, y \in L^{2}(\Delta, X)$; then

$$
\begin{gathered}
\int_{\Delta}\left\|\left(\delta_{e^{\prime}}(T-\lambda)^{1 / 2}-\delta_{\varepsilon}(T-\lambda)^{1 / 2}\right) x(\lambda)\right\|^{2} d \lambda \rightarrow 0 \quad\left(\varepsilon, \varepsilon^{\prime} \rightarrow 0\right), \\
\int_{\Delta \times \Delta^{\prime}}\left(\delta_{e}(T-\lambda) \delta_{e}(T-\nu) x(\nu), y(\lambda)\right) d \lambda d \nu \\
-\int_{\Delta \cap \Delta^{\prime}}\left(\delta_{e}(T-\lambda) x(\lambda), y(\lambda)\right) d \lambda \rightarrow 0 \quad(\varepsilon \rightarrow 0) .
\end{gathered}
$$

Proof OF Theorem III.2. (a) In virtue of Stone's theorem it suffices to prove that $(\varepsilon / \pi)\|R(\lambda+i \varepsilon) x\|^{2}$ for all $x \in Y$ is a Cauchy sequence in $\varepsilon$ in $L^{1}(\Delta)$. We have

$$
\frac{\varepsilon}{\pi}\|R(\lambda+i \varepsilon) x\|^{2}=\frac{\varepsilon}{\pi}\left\|R_{0}(\lambda+i \varepsilon) Q(\lambda+i \varepsilon) x\right\|^{2},
$$

where $Q(z)=(T-z)(H-z)^{-1}$. The term on the right-hand side is Cauchy in $\varepsilon$ in $L^{1}(\Delta)$ by $(1)$. 
(b) $W^{(e)}(\Delta)^{*}$ is bounded as follows from

$$
\begin{aligned}
\left\|W^{(e)}(\Delta)^{*} u\right\| & \leqslant\left\{\frac{|\varepsilon|}{\pi} \int_{\Delta}\left\|R_{0}(\lambda+i \varepsilon) Q(\lambda+i \varepsilon) u\right\|^{2} d \lambda\right\}^{1 / 2} \\
& \leqslant\|Q(\cdot+i \varepsilon) u\|_{L^{2}(\Delta, X)} .
\end{aligned}
$$

$W^{(e)}(\Delta)^{*}$ converges strongly as $\varepsilon \rightarrow \pm 0$ :

$$
\begin{aligned}
\| W^{\left(e^{\prime}\right)}(\Delta)^{*}- & W^{(e)}(\Delta)^{*} u \| \\
< & \left\{2 \int\left\|\left(\delta_{e^{\prime}}(T-\lambda)^{1 / 2}-\delta_{e}(T-\lambda)^{1 / 2}\right) Q(\lambda+i \varepsilon) u\right\|^{2} d \lambda\right\}^{1 / 2} \\
& +\left\{\int_{\Delta}\left\|\delta_{\varepsilon}(T-\lambda)\left(Q\left(\lambda+i \varepsilon^{\prime}\right)-Q(\lambda+i \varepsilon)\right) u\right\|^{2} d \lambda\right\}^{1 / 2} .
\end{aligned}
$$

The right-hand side converges to 0 as $\varepsilon, \varepsilon^{\prime} \rightarrow \pm 0$ by (i), (ii) and (1).

(c) Using (2),

$$
W^{(e)}(\Delta) W^{(e)}\left(\Delta^{\prime}\right)^{*}=\int_{\Delta \cap \Delta^{\prime}} Q(\lambda+i \varepsilon)^{*} \delta_{\varepsilon}(T-\lambda) Q(\lambda+i \varepsilon) d \lambda+o(1)
$$

weakly as $\varepsilon \rightarrow \pm 0$. Using the definition of $Q(z)$,

$$
\int_{\Delta} \delta_{\varepsilon}(H-\lambda) d \lambda=\int_{\Delta} Q(\lambda+i \varepsilon)^{*} \delta_{\varepsilon}(T-\lambda) Q(\lambda+i \varepsilon) d \lambda
$$

as a sesquilinear form on $Y$. Comparing these two equations we arrive at the desired relation.

Notes and literature comments.

\$I. Theorem I.2 is an abstractization of the main step of the spectral theorem for $N$-body systems [8]. Lemma I.3 originates in the commutator method of V. Enss [4]. A statement close to Corollary I.4 can be found in [3]. Note that $\sigma_{\text {ess }}(H) \subset$ $\cup \sigma\left(H_{a}\right)$ is a difficult direction of the famous HVZ theorem (see e.g. [15]).

§II. The first part of the section is a reformulation of Berezin's equations [2] studied in detail in [16], [17].

\$III. Lemma III.1 was proven in [19]. Theorems III.2 and III.4 are special cases of the corresponding theorems from [19] (see also [13], [23] and [20]). An earlier result of this genre was established in [11], [14] and [29]. Lemma III.5 was proven in [19]. A slightly weaker statement was proven earlier in [6 (see also [12]). The spaces $\Sigma A_{i} \mathcal{H C}$ of Lemma III.8 were introduced in [19] and in an earlier form in [17].

§IV. A statement similar to Proposition IV.10 was proven by G. Hagedorn [7].

§VI. Our $X$-operators are similar to those of Enss [4]. Proposition VI.1 is a simple exercise [15] on embedding theorems for Sobolev spaces and on compact integral operators. Proposition VI.2 belongs to T. Kato [11]. Proposition VI.3 was proven by G. Hagedorn [7] (see also [5], [19]) who has extended a close result of $R$. Iorio and M. O'Carroll [9]. Proposition VI.4 is a special case of a result of I. M. Sigal [17]. A similar result was obtained earlier by $\mathrm{K}$. Hepp [25].

A discussion of dilation analyticity as well as the Balslev-Combes theorems can be found in [15], [26] and [27]. 
Detailed references on asymptotic completeness theorems can be found in [19].

Theorem VI.8 is our main result. Note the analytic extension of the $N$-body $S$-matrix was proven for the first time by G. Hagedorn [6] for (two-cluster) $\rightarrow$ (twocluster) scattering. Our proof is completely different from that of G. Hagedorn. As in the two-particle scattering, he represents the integral kernels of the corresponding matrix elements of the $S$-matrix as sesquilinear forms and applies the Combes analytic continuation to those forms.

The analyticity of the diagonal elements of the $S$-matrix in the three body scattering and the many-body scattering below the three-body thresholds was proven by E. Balslev [1], [33].

$\S$ VII. See remarks to Proposition VI.3.

§VIII. See remarks to Proposition VI.4.

§IX. The scattering theory for nonselfadjoint operators was constructed in a different way by T. Kato [11] and Kako and K. Yajima [10] and applied by E. Balslev [1].

Appendix I. Results close to those of the appendix were announced in [18]. More general results were proved in [19]. Lemma AI.3 was proven by G. Hagedorn [7].

Appendix II. There is a simpler proof of a close theorem [11] using Kato's relative smoothness method. We stick to the given proven since it uses only resolvents and therefore is extendable onto the nonselfadjoint case.

\section{REFERENCES}

1. E. Balslev, Aarhus (preprint).

2. F. A. Berezin, Asymptotic behavior of eigenfunctions in Schrödinger's equation for many particles, Dokl. Akad. Nauk SSSR 163 (1965), 795-798.

3. P. Deift, W. Hunziker, B. Simon and E. Vock, Pointwise bounds on eigenfunctions and wave packets in N-body quantum systems. IV, Comm. Math. Phys. 64 (1978), 1-34.

4. V. Enss, A note on Hunziker's theorem, Comm. Math. Phys. 52 (1977), 233-238.

5. M. Combesqure and J. Ginibre, Hilbert space approach to the quantum mechanical three-body problem, Ann. Inst. H. Poincaré Sect. A 21 (1974), 97-145.

6. G. Hagedorn, $A$ link between scattering resonances and dilation analytic resonances in few body quantum mechanics, Comm. Math. Phys. 65 (1979), 181-188.

7. Asymptotic completeness for classes of two, three and four particle Schrödinger operators, Trans. Amer. Math. Soc. 258 (1980), 1-75.

8. W. Hunziker, On the spectra of Schrödinger multiparticle Hamiltonians, Helv. Phys. Acta 39 (1966), 451-462.

9. R. J. Iorio and M. O'Carroll, Asymptotic completeness for multiparticle Schrödinger operators with weak potentials, Comm. Math. Phys. 27 (1972), 137-145.

10. T. Kako and K. Yajima, Spectral and scattering theory for a class of non-self-adjoint operators, J. Math. Soc. Japan (to appear).

11. T. Kato, Wave operators and similarity for some nonself-adjoint operators, Math. Ann. 162 (1966), 258-279.

12. , Smooth operators and commutators, Stud. Math. 31 (1968), 535-546.

13. __ Two-space scattering theory, with applications to many-body problems, J. Fac. Sci. Univ. Tokyo Sect. IA Math. 24 (1977), 503-514.

14. T. Kato and S. T. Kuroda, The abstract theory of scattering, Rocky Mountain J. Math. 1 (1971), 127-171.

15. M. Reed and B. Simon, Methods of modern mathematical physics. IV, Academic Press, New York, 1978.

16. I. M. Sigal, On the discrete spectrum of the Schrödinger operators of multiparticle systems, Comm. Math. Phys. 48 (1976), 137-154. 
17. , Mathematical foundations of quantum scattering theory for multiparticle systems, Mem.

Amer. Math. Soc. No. 209 (1978).

18. On quantum mechanics of many-body systems with dilation-analytic potentials, Bull. Amer. Math. Soc. 84 (1978), 152-154.

19. Scattering theory for multiparticle systems. I, II, Preprint ETH-Zurich (1977-1978) (an expanded version will appear in Springer Lecture Notes in Math.).

20. __ On abstract scattering theory for two spaces, Princeton University (preprint).

21. __ Spectral theory of many-body Schrödinger operators, (unpublished).

22. E. Stein and G. Weiss, Introduction to Fourier analysis on Euclidean spaces, Princeton Univ. Press, Princeton, N. J., 1971.

23. K. Yajima, An abstract stationary approach to three-body scattering, J. Fac. Sci. Univ. Tokyo Sect. IA Math. 25 (1978), 109-132.

24. B. Simon, The theory of resonances for dilation-analytic potentials and the foundations of time dependent perturbation theory, Ann. of Math. (2) 97 (1973), 274-274.

25. K. Hepp, On the quantum mechanical N-body problem, Helv. Phys. Acta. 42 (1969), 425-458.

26. D. Babbitt and E. Balslev, Dilation-analyticity and decay properties of interactions, Comm. Math. Phys. 35 (1974), 173-179.

27. _ A characterization of the dilation-analytic potentials and vectors, J. Funct. Anal. 18 (1974), $1-14$.

28. E. Balslev and J. M. Combes, Spectral properties of many-body Schrödinger operators with dilation-analytic interactions, Comm. Math. Phys. 22 (1971), 280-294.

29. T. Kato and S. T. Kuroda, Theory of simple scattering and eigenfunction expansions, Functional Analysis and Related Fields (edited by F. Browder), Springer-Verlag, Berlin and New York, 1970.

30. R. Lavine, Commutators and scattering theory, Comm. Math. Phys. 20 (1971), 301-323.

31. __ Absolute continuity of positive spectrum for Schrödinger operators with long-range potentials,

J. Funct. Anal. 12 (1973), 30-54.

32. E. Mourre, Applications de la méthode de Lavine ou problème a trois corps.

33. E. Balslev, Analytic scattering theory for many-body systems below the smallest three-body threshold, Aarhus (preprint).

34. J. Weidman, The virial theorem and its application to the spectral theory of Schrödinger operators, Bull. Amer. Math. Soc. 73 (1967), 452-456.

35. I. M. Sigal, $A$ remark on the absence of bound states in the many-body systems (in preparation).

36. B. Simon, Absence of positive eigenvalues in a class of multiparticle quantum systems, Math. Ann. 207 (1974), 133-138.

37. E. Balslev, Absence of positive eigenvalues of Schrödinger operators, Arch. Rational Mech. Anal. 59 (1975), 343-357.

Department of Mathematics, Princeton University, Princeton, New Jersey 08544

Current address: Department of Theoretical Mathematics, The Weizmann Institute of Science, Rehovot 76100, Israel 